\title{
Algebraic Differential Spatial Modulation is Capable of Approaching the Performance of its Coherent Counterpart
}

\author{
Rakshith Rajashekar, Chao Xu, Member, IEEE, Naoki Ishikawa, Student Member, IEEE, \\ Shinya Sugiura, Senior Member, IEEE, K.V.S. Hari, Fellow, IEEE, and L. Hanzo, Fellow, IEEE
}

\begin{abstract}
We show that certain signal constellations invoked for classic differential encoding result in a phenomenon we term as the unbounded differential constellation size (UDCS). Various existing differential transmission schemes that suffer from this issue are identified. Then, we propose an enhanced algebraic field extension based differential spatial modulation scheme (AFEDSM) and its enhanced counterpart that strikes a diversity-rate trade-off (AFE-DSM-DR), both of which overcome the UDCS issue without compromising its full transmit diversity advantage. Furthermore, the proposed schemes are extended to incorporate amplitude and phase shift keying (APSK) in order to exploit all the available degrees of freedom. Additionally, we propose a pair of detection schemes specially designed for APSK aided differential transmission schemes. Explicitly, we conceive the buffered minimum mean squared error (B-MMSE) detector and buffered maximum likelihood (B-ML) detector, which exploit the knowledge of previously detected symbols in order to further improve the detection performance. Our simulation results have shown that the proposed detectors are capable of bridging the performance gap between the conventional differential detector (CDD) and the coherent detector that has full channel state information. Specifically, when employing the proposed APSK aided AFE-DSM scheme operating at a rate of 2 bits per channel use (bpcu), the B-MMSE and B-ML detectors are observed to give about $3 \mathrm{~dB}$ and $3.5 \mathrm{~dB}$ signal-to-noise ratio gain with respect to their CDD counterpart at a bit error ratio of $10^{-5}$.
\end{abstract}

Index Terms-Differential spatial modulation, finite input constellation, diversity, minimum mean squared error, maximum likelihood detector.

\section{INTRODUCTION}

The employment of multiple-input multiple-output (MIMO) systems is imperative for achieving a high spectral efficiency

R. Rajashekar, C. Xu and L. Hanzo are with the School of ECS, University of Southampton, UK (e-mail: rmr1u14@soton.ac.uk, cx1g08@ecs.soton.ac.uk, 1h@ecs.soton.ac.uk).

K.V.S. Hari is with the Dept. of ECE, Indian Institute of Science, Bangalore, India (e-mail: hari@ece.iisc.ernet.in).

N. Ishikawa is with the Graduate School of Information Sciences, Hiroshima City University, Hiroshima 731-3194, Japan (e-mail: naoki@ishikawa.cc).

S. Sugiura is with the Department of Computer and Information Sciences, Tokyo University of Agriculture and Technology, Koganei 184-8588, Japan (e-mail: sugiura@ieee.org).

The financial support of the EPSRC projects EP/N004558/1 and EP/L018659/1, as well as of the European Research Council's Advanced Fellow Grant under the Beam-Me-Up project and of the Royal Society's Wolfson Research Merit Award is gratefully acknowledged.

The work of N. Ishikawa was partially supported by the SCAT fellowship and the MEXT/JASSO Tobitate fellowship of Japan.

The work of S. Sugiura was supported in part by the Japan Society for the Promotion of Science (JSPS) KAKENHI Grant Number 16KK0120, which is gratefully acknowledged.
[1], [2]. While MIMO systems support high data rates, they suffer from low energy efficiency [3], [4] owing to the power hungry RF-front end components, such as amplifiers and mixers that are required for each of the transmit and receive chains. Furthermore, multi-stream transmissions [2] suffer from a high maximum likelihood (ML) detection complexity at the receiver. Some of these issues were addressed by the recent single-RF chain based transmission scheme known as spatial modulation [5]-[8], which supports low-complexity single stream ML detection [9]-[13] and it is also energy efficient [14], [15]. Specifically, the SM system activates only a single transmit antenna (TA) at any time instant, thus a single transmit RF chain is sufficient [5]. More specifically, in SM the input bitstream is divided into blocks of $\log _{2}\left(M N_{t}\right)$ bits, and in each block $\log _{2}\left(N_{t}\right)$ bits are used for activating a TA from $N_{t}$ transmit antennas and $\log _{2}(M)$ bits are used to select a symbol from an $M$-ary alphabet.

While the SM system offers several advantages over conventional multistream MIMO [2], it suffers from the lack of transmit diversity owing to its single TA activation constraint. This issue was addressed by amalgamating space-time block codes (STBC) and the SM scheme [16]-[20]. Furthermore, closed loop schemes were conceived for further addressing this issue, which include link adaptation based SM [21], [22], and antenna subset selection aided SM [23]-[26]. However, the majority of the literature, including the family of closed loop techniques mentioned above, was designed for coherent communication, where accurate channel impulse response (CIR) estimation and tracking are assumed at the receiver [27]. But attaining accurate CIRs imposes significant training overhead [27] and hence renders coherent communication unsuitable for rapidly fading high-doppler channel conditions. Differential encoding [28], [29] overcomes this issue, where each transmitted symbol is a function of the source symbol to be transmitted as well as of the previous transmitted symbol.

Hence, there has been a significant research interest in SM operating in non-coherent communication scenarios [30]-[38], which is commonly referred to as differential SM (DSM). Specifically, the authors of [30] have proposed permutation matrix based DSM (P-DSM), which uses one of the $\left(N_{t} \times N_{t}\right)$ permutation matrices as the dispersion matrix (DM). In [31], the authors have proposed a fixed set of sparse complex-valued matrices to be used as DMs, which is referred to as the DM based DSM (DM-DSM). In [32], the authors have proposed a cyclic signal structure based DSM scheme (CS-DSM), which 
was specifically meant for two transmit antennas. In [33], the authors have proposed a field extension based DSM scheme (FE-DSM) as well as a FE-DSM scheme that is capable of striking a flexible trade-off between the diversity order and transmission rate (FE-DSM-DR). More recently, the authors of [34], [35] have proposed an amplitude and phase shift keying (APSK) aided P-DSM scheme, which is referred to as APSK-DSM. Note that all the aforementioned schemes use either PSK or APSK signal sets, and have only one nonzero element in each row and column of the transmit spacetime matrix (STM). Furthermore, when employing APSK signal sets, the transmit STM is non-unitary and hence the conventional differential detector (CDD) becomes suboptimal. In [50], the authors have proposed a minimum mean squared error (MMSE) detector that performs better than the CDD when employing a non-orthogonal space-time block coded differential transmission scheme (D-STBC). It is also worth mentioning that the differential input constellation of DMDSM, FE-DSM, FE-DSM-DR and D-STBC schemes grows unbounded with the transmission block owing to the differential encoding, which we refer to as having unbounded differential constellation size (UDCS). This problem usually arises, when the PSK constellation employed has an arbitrary tilt or when more than one TAs are activated during a given channel use, as in the case of D-STBC. Note that the unbounded constellation issue makes the employment of a high-resolution digital-to-analog convertor (DAC) imperative, which is both expensive and power hungry. On the other hand, if the differential constellation is designed so that it is free from the UDCS issue, then low-cost amplifiers and phase shifters would be sufficient to implement the transmitter, hence alleviating the need for expensive DACs. We discuss this issue in detail in Section-II-C. Table I summarizes the various characteristics of the differential transmission schemes discussed so far.

Against this background, the new contributions of this paper are:

1) We first provide a formal discussion of the UDCS issue ${ }^{1}$ and then study the existing schemes in this new light. Our theoretical analysis shows that the existing FEDSM and FE-DSM-DR schemes, which rely on transcendental field extensions [33], suffer from the UDCS issue. Furthermore, we propose novel algebraic field extension based FE-DSM and FE-DSM-DR schemes, which overcome this issue without compromising the full transmit diversity advantage and low-complexity ML detection. The proposed algebraic field extension based schemes are termed as the AFE-DSM and AFEDSM-DR schemes, which have the following benefits:

a) The proposed schemes are amenable for lowcomplexity differential encoding, where we do not have to multiply complex-valued matrices, instead we obtain the multiplied matrix from a pre-defined dictionary.

b) Since the proposed schemes do not suffer from the

\footnotetext{
${ }^{1}$ Note that the UDCS issue is also studied in [40], though not in the same context and depth as in the present paper.
}

UDCS issue, they do not require a high resolution DAC at the transmitter [39], and hence they facilitate low-cost and low-power system design.

c) Unlike the existing FE-DSM-DR scheme, which requires two phase angles to be optimized by computer search, the proposed AFE-DSM-DR scheme systematically determines these phases.

Furthermore, in order to achieve higher rates, we further extend the AFE-DSM and AFE-DSM-DR schemes by considering APSK constellations with the aid of differential amplitude encoding. It is worth mentioning that the number of ring amplitudes $L_{a}$ in the proposed APSK extensions are generic unlike those of [34] and [35]. Furthermore, we employ absolute amplitude encoding in the proposed extensions in contrast to their differential counterparts proposed in [34] and [35], owing to their ability achieve higher capacity as observed in [41].

2) The APSK aided differential transmission schemes result in non-unitary transmit STMs, which render the CDD suboptimal. The MMSE detector of [50] is known to achieve better performance than the CDD in case of differential schemes employing non-unitary transmit STMs. In this paper, we further generalize the MMSE detector of [50] and propose a novel buffered MMSE (B-MMSE) detector, which makes use of the previously detected transmit STMs. Note that the search complexity of the proposed B-MMSE detector remains the same as that of the CDD.

3) We propose a novel buffered maximum likelihood (BML) detector for differential transmission schemes employing non-unitary STMs. As in the case of the BMMSE detector, the proposed B-ML detector makes use of the previously detected transmit STMs in order to improve the detection performance and its search complexity remains the same as that of the CDD. Furthermore, the B-ML detector is observed to bridge the performance gap between the coherent and noncoherent communication schemes, thus making it an attractive solution.

The rest of the paper is organized as follows. Section II details the system model of DSM and discusses the fundamental issues in differential encoding. In Section III, the proposed AFE-DSM and AFE-DSM-DR schemes are presented. In Section IV-A we extend the AFE-DSM and AFE-DSM-DR schemes to support APSK signaling with the aid of differential amplitude encoding. Furthermore, the novel B-MMSE and BML detectors are presented in Section IV-B and Section IV-C, respectively. Section $\mathrm{V}$ provides our simulation results, while 
TABLE I

COMPARISON OF VARIOUS EXISTING DIFFERENTIAL TRANSMISSION SCHEMES.

\begin{tabular}{|c|c|c|c|c|c|}
\hline & $\begin{array}{c}\text { No. of transmit } \\
\text { RF chains } \\
\text { required }\end{array}$ & $\begin{array}{l}\text { Throughput } \\
\text { (bpcu) }\end{array}$ & $\begin{array}{l}\text { Achievable diversity } \\
\text { order }\end{array}$ & $\begin{array}{c}\text { Number of } \\
\text { transmit antennas } \\
\text { supported }\end{array}$ & $\begin{array}{c}\text { Is input } \\
\text { constellation size } \\
\text { bounded ? }\end{array}$ \\
\hline P-DSM [30] & 1 & $\frac{\log _{2}\left(M^{N_{t}}\right)+\log _{2}\left\lfloor\left(N_{t} !\right)\right\rfloor_{2} p}{N_{t}}$ & $N_{r}$ & $N_{t}$ & Yes \\
\hline DM-DSM [31] & 1 & $\frac{\log _{2}\left(M^{N_{t} / d}\right)+\log _{2}(Q)}{N_{t}}$ & $\begin{array}{c}d N_{r} \\
\left(1 \leq d \leq N_{t}\right)\end{array}$ & $N_{t}$ & No \\
\hline CS-DSM [32] & 1 & $\frac{\log _{2}\left(Q^{\prime} Q\right)}{2}$ & $2 N_{r}$ & 2 & Yes \\
\hline FE-DSM [33] & 1 & $\frac{\log _{2}\left(M N_{t}\right)}{N_{t}}$ & $N_{t} N_{r}$ & $N_{t}$ & No \\
\hline FE-DSM-DR [33] & 1 & $\frac{h \log _{2}(M g)+\log _{2}(h)}{N_{t}}$ & $\begin{array}{c}g N_{r}<N_{t} N_{r} \\
\left(g=N_{t} / h\right)\end{array}$ & $N_{t}$ & No \\
\hline $\begin{array}{l}\text { APSK-DSM [34] } \\
\text { (1-bit ASK) }\end{array}$ & 1 & $\frac{\log _{2}\left(M^{N_{t}}\right)+\log _{2}\left\lfloor\left(N_{t} !\right)\right\rfloor_{2} p+N_{t}}{N_{t}}$ & $N_{r}$ & $N_{t}$ & Yes \\
\hline $\begin{array}{l}\text { APSK-DSM [35] } \\
\text { (2-bit ASK) }\end{array}$ & 1 & $\frac{\log _{2}\left(M^{N_{t}}\right)+\log _{2}\left\lfloor\left(N_{t} !\right)\right\rfloor_{2} p+2 N_{t}}{N_{t}}$ & $N_{r}$ & $N_{t}$ & Yes \\
\hline $\begin{array}{l}\text { D-STBC [50] } \\
\text { with STBCs } \\
\text { from [43], [47]-[49] }\end{array}$ & $N_{t}$ & $N_{t} \log _{2}(M)$ & $N_{t} N_{r}$ & $N_{t}^{\dagger}$ & No \\
\hline
\end{tabular}

bpcu : bits per channel use

$\lfloor c\rfloor_{2^{p}}$ denotes the largest integer that is a power of two and smaller than $c$

$Q:$ No. of DMs in [31], [32]

$d:$ transmit diversity order

$Q^{\prime}$ : No. of cyclic signal matrices in [32]

$\dagger$ Depends on the STBC employed

Section VI concludes the paper. $^{2}$

\section{Differential Spatial Modulation System}

Consider a MIMO system having $N_{t}$ TAs and $N_{r}$ RAs operating in a quasi-static Rayleigh flat-fading channel, where each of the channel realizations remains constant for several successive channel uses, which is modeled as

$$
\mathbf{Y}_{i}=\sqrt{\rho} \mathbf{H}_{i} \mathbf{X}_{i}+\mathbf{N}_{i}
$$

where $\mathbf{H}_{i} \in \mathbb{C}^{N_{r} \times N_{t}}$ and $\mathbf{N}_{i} \in \mathbb{C}^{N_{r} \times N_{t}}$ are the channel and noise matrices, respectively, whose entries are from $\mathcal{C N}(0,1)$, $\mathbf{X}_{i} \in \mathbb{C}^{N_{t} \times N_{t}}$ is the transmitted space-time matrix (STM), $\mathbf{Y}_{i} \in \mathbb{C}^{N_{r} \times N_{t}}$ is the received STM, and $\rho$ denotes the average signal-to-noise ratio (SNR) at each receive antenna. The subscript $i$ in all matrices indicates the block index.

${ }^{2}$ Notations: Uppercase and lowercase boldface letters represent matrices and vectors, respectively. If $Q_{1}$ and $Q_{2}$ are two sets, then $Q_{3}=Q_{1} \times Q_{2}$ represents the Cartesian product of the sets $Q_{1}$ and $Q_{2}$. Furthermore, $\|\cdot\|$ represents the Frobenius norm of a matrix, or the two-norm of a vector. The notations of $(\cdot)^{H}$ and $(\cdot)^{T}$ indicate the Hermitian transpose and transpose of a vector/matrix, respectively, while $|\cdot|$ represents the magnitude of a complex quantity, or the cardinality of a given set. $\mathbb{C}$ and $\mathbb{R}$ represent the field of complex and real numbers, respectively. If $\mathbf{y}$ is an $m$ length vector, then $\operatorname{diag}(\mathbf{y})$ represents an $m \times m$ diagonal matrix whose $(j, j)^{\text {th }}$ element is $\mathbf{y}_{j}$. Furthermore, $\otimes$ defines the Kronecker product of two matrices. $\mathcal{C N}\left(\beta, \sigma^{2}\right)$ denotes a complex Gaussian random variable with mean $\beta$ and variance $\sigma^{2}$. If $L$ is a field, then $L[X]$ represents the ring of polynomials in $X$ over $L$.

\section{A. DSM System}

If $\mathbf{S}_{i} \in \mathbb{C}^{N_{t} \times N_{t}}$ represents the unitary STM to be transmitted during the symbol period of the $i^{\text {th }}$ block, then the differential encoding [28], [29] of the transmitted STM is represented by $\mathbf{X}_{i}=\mathbf{X}_{i-1} \mathbf{S}_{i}$, where $\mathbf{X}_{0}$ is taken to be $\mathbf{I}_{N_{t}}$. Since the SM system employs only a single RF chain at the transmitter, each column of $\mathbf{S}_{i}$ is assumed to have only a single non-zero element. Assuming that the channel remains constant over a period of two successive blocks, we have $\mathbf{Y}_{i-1}=\sqrt{\rho} \mathbf{H}_{i} \mathbf{X}_{i-1}+\mathbf{N}_{i-1}$, and hence (1) can be written as $\mathbf{Y}_{i}=\mathbf{Y}_{i-1} \mathbf{S}_{i}+\mathbf{N}_{i}-\mathbf{N}_{i-1} \mathbf{S}_{i}$. Assuming that there is no channel state information (CSI) at the receiver, the optimal differential detector [28] is given by

$$
\hat{\mathbf{S}}_{i}=\arg \min _{\mathbf{S} \in \mathcal{S}}\left\|\mathbf{Y}_{i}-\mathbf{Y}_{i-1} \mathbf{S}\right\|^{2},
$$

where $\mathcal{S}$ is the set of transmit STMs. Note that the detector in (2) is optimal when the transmit STMs are unitary [29]. In case of non-unitary STMs, such as APSK aided DSM schemes, novel optimal detectors are to be conceived.

\section{B. Dispersion-Matrix Based DSM [33]}

The transmitted STM in case of DM-DSM is of the form $\mathbf{S}_{i}=\mathbf{D}(\mathbf{s}) \mathbf{A}_{q}$, where we have $\mathbf{D}(\mathbf{s}) \in \mathcal{D}=$ $\left\{\operatorname{diag}(\mathbf{s}) \mid s_{i} \in \mathcal{L}_{i}-\mathrm{PSK}\right\}, \mathbf{s}=\left[s_{1}, s_{2}, \ldots, s_{N_{t}}\right]$, and $\mathbf{A}_{q} \in \mathcal{A}$, 
where $\mathcal{A}=\left[\mathbf{A}_{1}, \mathbf{A}_{2}, \ldots, \mathbf{A}_{Q}\right]$ is the set of DMs. The achievable transmission rate of the DM-DSM is given by $R_{D M-D S M}=\frac{\log _{2}\left(Q \cdot \mathcal{L}_{1} \cdots \mathcal{L}_{N_{t}}\right)}{N_{t}}$ bpcu.

C1: It is required that each element of $\mathcal{A}$ should be a unitary matrix [29] and should have only a single non-zero element in each column and row. The latter condition is necessary, since the SM system can transmit only a single symbol in each channel-use owing to using a single RF chain at the transmitter.

\section{A Fundamental Issue in Differential Encoding}

Considering the differentially encoded STM at the $i^{\text {th }}$ block, we have

$$
\begin{aligned}
\mathbf{X}_{i}= & \mathbf{X}_{i-1} \mathbf{S}_{i}, \\
= & \mathbf{X}_{i-2} \mathbf{S}_{i-1} \mathbf{S}_{i}, \\
& \vdots \\
= & \mathbf{X}_{0} \mathbf{S}_{1} \ldots \mathbf{S}_{i-2} \mathbf{S}_{i-1} \mathbf{S}_{i},
\end{aligned}
$$

which is essentially the product of all the STMs transmitted from the first channel use, since we have $\mathbf{X}_{0}=\mathbf{I}_{N_{t}}$. Since $\mathbf{S}_{k} \in \mathcal{S}$ for $1 \leq k \leq i$, we have $\mathbf{X}_{i} \in \mathcal{S}_{i}=\underbrace{\mathcal{S} \times \mathcal{S} \times \cdots \times \mathcal{S}}_{i \text { times }}$ and $\left|\mathcal{S}_{i}\right|=|\mathcal{S}|^{i}$. Thus, the signal set that the transmitter has to support grows exponentially with the number of transmission blocks, unless the set $\mathcal{S}$ is designed to satisfy certain specific conditions. Since practical systems operate at a finite transmission power, the signal set $\mathcal{S}_{i}$ would become dense for large $i$, which renders the scheme unsuitable for practical use. Let us illustrate this issue by considering a simple singlesymbol differential transmission scheme, where a differentially encoded symbol $x_{i}=x_{i-1} s_{i}$ is transmitted at the $i^{\text {th }}$ time instant analogous to (3). Let us consider two $M$-PSK signal sets $S=\left\{e^{j 2 \pi k / M}\right\}_{k=0}^{M-1}$ and $S^{\prime}=\left\{e^{j(2 \pi k / M+\theta)}\right\}_{k=0}^{M-1}=e^{j \theta} S$, where $S^{\prime}$ is the same as $S$, except for the phase offset $\theta$. The corresponding differential signal sets of the $i^{\text {th }}$ transmission block are $S_{i}=\underbrace{S \times S \times \cdots \times S}_{i \text { times }}$ and $S_{i}^{\prime}=\underbrace{S^{\prime} \times S^{\prime} \times \cdots \times S^{\prime}}_{i \text { times }}$. Since $S$ is closed under multiplication, it can be easily shown that $S_{i}=S$ and

$$
S_{i}^{\prime}=\left\{e^{j(2 \pi / M) \sum_{l=1}^{i} k_{l}} e^{j(i \theta)}\right\}_{\left\{k_{l}\right\}_{l=1}^{i}=0}^{M-1} .
$$

Lemma 1: If $\theta$ is any algebraic real number such that $e^{j \theta}$ is transcendental, then $\bigcap_{l=1}^{i} S_{l}^{\prime}$ is a null set for any finite $i \geq 2$.

Proof: Please refer to Appendix A.

We can infer from Lemma 1 and (6) that each of the differential signal sets $S_{i}^{\prime}$ is distinct and hence the number of constellation points to be supported by the transmitter grows unbounded with the number of transmission blocks $i$. In the aforementioned case, the transmitter should support nearly every phase value in the set $[0,2 \pi)$, which practical phase shifters can hardly support. Again, this issue is termed as the problem of unbounded differential constellation size. By contrast, the set $S$ does not result in this issue. Thus, the choice of phase offset used in the signal set plays a crucial role in the differential encoding scheme.
Let us now focus our attention on the the existing DSM schemes [30]-[33]. It can be readily verified that the P-DSM [30] and CS-DSM [32] schemes do not suffer from the UDCS, while the DM-DSM [31], FE-DSM and FE-DSM-DR [33] do suffer from this issue. More specifically, the FE-DSM and FE-DSM-DR [33] employ transcendental elements chosen from the unit circle as phase offset of the PSK signal set, while the DM-DSM scheme employs arbitrary elements which are chosen from the unit-circle as the phase offset of the PSK signal set. Thus, the schemes in [31] and [33] pose the issue of UDCS. Furthermore, it is worth mentioning that the UDCS issue highlighted above can be seen in other differential transmission schemes in the literature, which employ

1) orthogonal space-time block codes [45], [46] combined with QAM signal sets,

2) non-orthogonal STBCs constructed both from division algebras [43] and from number theory [47], the Golden code [48] and the perfect STBCs [49].

The performance of these STBCs in the context of differential transmission scheme can be found in [50]. In case of the aforementioned STBCs, the differential constellation becomes dense both in phase as well as in amplitude, when the number of transmission blocks is increased.

\section{Proposed Algebraic Field Extension Based Dispersion Matrix Set Constructions}

A brief overview of algebraic field extensions as required for our exposition on the proposed DM set construction can be found in Section III-A of [33]. For further details, the readers are referred to [42], [43]. In this section, we first provide a brief overview of the FE-DSM and FE-DSM-DR schemes [33], which were constructed based on transcendental field extensions, and then discuss the UDCS issue in the context of these schemes. Secondly, we propose algebraic field extension based DSM schemes for overcoming the UDCS issue, while retaining all the benefits of the FE-DSM and FE-DSM-DR schemes. The proposed schemes are referred to as AFE-DSM and AFE-DSM-DR, which are the algebraic counterparts of FE-DSM and FE-DSM-DR, respectively.

\section{A. FE-DSM and FE-DSM-DR [33]}

1) FE-DSM: Let $S=\left\{\omega_{M}^{i}\right\}_{i=0}^{M-1}$ represent the conventional $M$-PSK signal set, where $\omega_{M}=e^{j 2 \pi / M}$. Let $u_{1}$ be algebraic over $\mathbb{Q}$ and $a_{0}=-e^{j u_{1}}$ be a transcendental element over $\mathbb{Q}(S)$. For example, $u_{1}$ can be $\sqrt{3}$, which is a root of the polynomial $x^{2}-3$. Consider the field $F=\mathbb{Q}\left(S, e^{j u_{1}}\right)$, where the polynomial $x^{n}+a_{0}=x^{n}-e^{j u_{1}}$ (for any $n$ ) is irreducible over $F$. Furthermore, consider the extended field $K=F(\alpha)$, where $\alpha$ is the primitive $n^{\text {th }}$ root of $e^{j u_{1}}$. The companion matrix associated with the primitive element is given by

$$
\mathbf{M}=\left[\begin{array}{ccccc}
0 & 0 & \cdots & 0 & e^{j u_{1}} \\
1 & 0 & \cdots & 0 & 0 \\
0 & 1 & \cdots & 0 & 0 \\
\vdots & \vdots & \ddots & \vdots & \vdots \\
0 & 0 & \cdots & 1 & 0
\end{array}\right] \in F^{n \times n} .
$$

The DM set used by the FE-DSM scheme is given by $\mathcal{A}=$ $\left\{\mathbf{I}_{n}, \mathbf{M}, \mathbf{M}^{2}, \ldots, \mathbf{M}^{n-1}\right\}$, where $n$ is chosen to be $N_{t}$. The 
transmit STM set is given by $\mathcal{S}=\mathcal{D} \times \mathcal{A}$, where the set $\mathcal{D}$ has scaled identity matrices of the form $s \mathbf{I}_{n}$, such that $s \in S$.

Example 1: Consider $n=N_{t}=3$ and $a_{0}=-e^{j \sqrt{2}}$. Then, the elements of set $\mathcal{A}$ are given by $\mathbf{I}_{3}$,

$$
\mathbf{M}=\left[\begin{array}{ccc}
0 & 0 & e^{j \sqrt{2}} \\
1 & 0 & 0 \\
0 & 1 & 0
\end{array}\right] \text { and } \mathbf{M}^{2}=\left[\begin{array}{ccc}
0 & e^{j \sqrt{2}} & 0 \\
0 & 0 & e^{j \sqrt{2}} \\
1 & 0 & 0
\end{array}\right] .
$$

The following proposition illustrates the UDCS issue in the FE-DSM scheme.

Proposition 1: In FE-DSM scheme, the set of differentially encoded input constellation points can grow as fast as $q M$ symbols over $i$ transmission blocks, where $q$ is the largest positive integer satisfying $i(n-1)=q n+r$ for some $0 \leq$ $r<n-1$.

Proof: Please refer to Appendix B.

2) FE-DSM-DR: Let $g \cdot h$ represent factors of $N_{t}$. A DM set that allows us to transmit $h$ independent $M$-PSK symbols in each transmit STM and achieve a transmit diversity order $g$ is constructed as follows. Considering $F=\mathbb{Q}\left(S,-e^{j u_{1}}\right)$ as before and the extension $K=F(\alpha)$, where $\alpha$ is a primitive $g^{\text {th }}$ root of the polynomial $p_{1}(x)=x^{g}-e^{j u_{1}}$, we obtain the DM set given by $\mathcal{A}^{\prime}=\left\{\mathbf{I}_{g}, \mathbf{M}, \mathbf{M}^{2}, \ldots, \mathbf{M}^{g-1}\right\}$, where $\mathbf{M} \in$ $F^{g \times g}$ is the companion matrix of $p_{1}(x)$. We define $\mathcal{D}$ to be a set of block-diagonal matrices given by

$\mathcal{D}=\left\{\operatorname{diag}\left(s_{1} \mathbf{A}_{1}, s_{2} \mathbf{A}_{2}, \ldots, s_{h} \mathbf{A}_{h}\right) \mid s_{i} \in M-\mathrm{PSK}, \mathbf{A}_{i} \in \mathcal{A}^{\prime}\right\}$

Let us now consider the field extension $L=K(\beta)$ associated with the polynomial $p_{2}(x)=x^{h}-e^{j u_{2}}$, where $e^{j u_{2}}$ is transcendental over $K$ and $\beta$ is the primitive $h^{\text {th }}$ root of $e^{j u_{2}}$. Then, the regular representation of an element $l=\sum_{i=0}^{h-1} k_{i} \beta^{i} \in L$ is given by $\sum_{i=0}^{h-1} k_{i} \mathbf{N}^{i}$, where $k_{i} \in K, 0 \leq i \leq h-1$ and $\mathbf{N} \in K^{h \times h}$ is the companion matrix of $p_{2}(x)$. We define the $\mathrm{DM}$ set as

$$
\mathcal{A}=\left\{\mathbf{I}_{n}, \mathbf{N}^{\prime}, \mathbf{N}^{\prime 2}, \ldots, \mathbf{N}^{\prime h-1}\right\},
$$

where $\mathbf{N}^{\prime}=\mathbf{N} \otimes \mathbf{I}_{g}$. The transmit STM set is given by $\mathcal{S}=$ $\mathcal{D} \times \mathcal{A}$ as before.

Example 2: Let $n=N_{t}=4, g=h=2, u_{1}=\sqrt{3}$ and $u_{2}=\sqrt{5}$. The elements of the set $\mathcal{D}$ are

$$
\begin{gathered}
{\left[\begin{array}{cccc}
s_{1} & 0 & 0 & 0 \\
0 & s_{1} & 0 & 0 \\
0 & 0 & s_{2} & 0 \\
0 & 0 & 0 & s_{2}
\end{array}\right],\left[\begin{array}{cccc}
0 & s_{1} e^{j \sqrt{3}} & 0 & 0 \\
s_{1} & 0 & 0 & 0 \\
0 & 0 & s_{2} & 0 \\
0 & 0 & 0 & s_{2}
\end{array}\right],} \\
{\left[\begin{array}{cccc}
0 & s_{1} e^{j \sqrt{3}} & 0 & 0 \\
s_{1} & 0 & 0 & 0 \\
0 & 0 & 0 & s_{2} e^{j \sqrt{3}} \\
0 & 0 & s_{2} & 0
\end{array}\right],\left[\begin{array}{cccc}
s_{1} & 0 & 0 & 0 \\
0 & s_{1} & 0 & 0 \\
0 & 0 & 0 & s_{2} e^{j \sqrt{3}} \\
0 & 0 & s_{2} & 0
\end{array}\right],}
\end{gathered}
$$

where $s_{1}$ and $s_{2}$ are from the classic $M$-PSK signal set. The elements of the DM set $\mathcal{A}$ are

$$
\left[\begin{array}{llll}
1 & 0 & 0 & 0 \\
0 & 1 & 0 & 0 \\
0 & 0 & 1 & 0 \\
0 & 0 & 0 & 1
\end{array}\right],\left[\begin{array}{cccc}
0 & 0 & e^{j \sqrt{5}} & 0 \\
0 & 0 & 0 & e^{j \sqrt{5}} \\
1 & 0 & 0 & 0 \\
0 & 1 & 0 & 0
\end{array}\right] .
$$
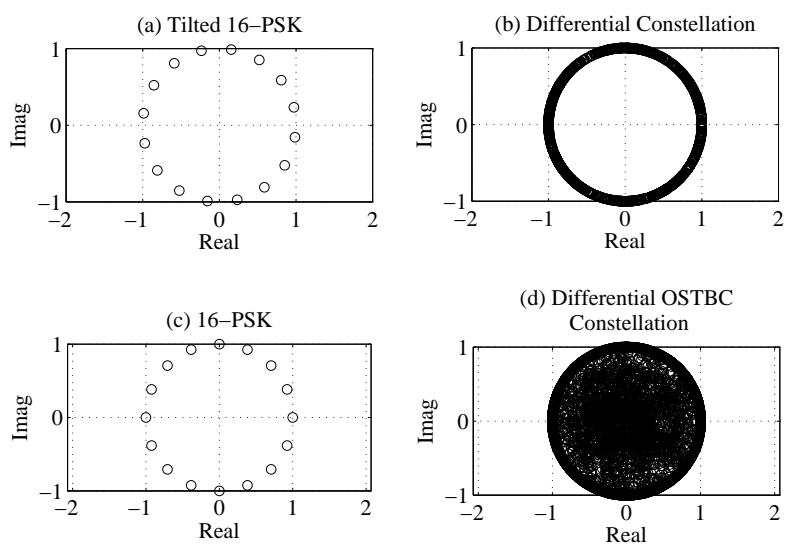

Fig. 1. Comparison of the coherent and differential signal constellations. Plot(a) and Plot(b) correspond to the tilted 16-PSK signal set and its differential counterpart, respectively. Plot(c) and $\operatorname{Plot}(\mathrm{d})$ correspond to the untilted 16-PSK and the resulting differential constellation when employing OSTBC, respectively. The differential constellation points correspond to the input constellation over thousand encoding blocks.

Since FE-DSM-DR uses FE-DSM as its diagonal blocks, it is easy to see that the differential constellation in case of FE-DSM-DR also grows unbounded and hence suffers from the UDCS issue. Fig. 1 illustrates the growth of the differential constellation size in case of transmission schemes employing tilted PSK signal in DM-DSM, FE-DSM, FEDSM-DR ${ }^{3}$, and in case of OSTBC employing untilted PSK signal set. Note that the differential constellation in case of OSTBC is normalized for ease of presentation. It is plausible that the transmitter in case of a differential scheme has to support a large set of constellation points. Specifically, the differential constellations of DM-DSM, FE-DSM, FE-DSMDR require nearly continuous phase, whereas in case of OSTBC aided differential transmission both the amplitude and phase are continuous. In the following section, we propose algebraic field extension based DM set constructions, which overcome the UDCS issue in contrast to the transcendental field extensions considered in [33].

\section{B. Proposed AFE-DSM and AFE-DSM-DR}

1) AFE-DSM: Let $S=\left\{\omega_{M}^{i}\right\}_{i=0}^{M-1}$ represent the conventional $M$-PSK signal set, where $\omega_{M}=e^{j 2 \pi / M}$. Consider a polynomial of the form $x^{n}+a_{0}$, where $a_{0}$ is chosen for ensuring that the polynomial is irreducible over $\mathbb{Q}(S)$. From Proposition 5 of [43], we show that $x^{n}+a_{0}$ is irreducible over $F=\mathbb{Q}(S)$ if $a_{0}=-\omega_{M}$, so that the primes in the prime factorization of $n$ forms a subset of the primes that appear in the prime factorization of $M$. Let us now consider the extended field $K=F(\alpha)$, where $\alpha$ is the primitive $n^{\text {th }}$ root of $\omega_{M}$. The companion matrix associated with the primitive element $\alpha$ is

\footnotetext{
${ }^{3}$ The tilted PSK signal points result from the arbitrary phase angles used in the DMs in case of DM-DSM and from the phase factors $\left(e^{j u_{1}}, e^{j u_{2}}\right)$ in case of FE-DSM and FE-DSM-DR schemes.
} 
given by

$$
\mathbf{M}=\left[\begin{array}{ccccc}
0 & 0 & \cdots & 0 & e^{j 2 \pi / M} \\
1 & 0 & \cdots & 0 & 0 \\
0 & 1 & \cdots & 0 & 0 \\
\vdots & \vdots & \ddots & \vdots & \vdots \\
0 & 0 & \cdots & 1 & 0
\end{array}\right] \in F^{n \times n},
$$

and the DM set used by the AFE-DSM scheme is given by $\mathcal{A}=\left\{\mathbf{I}_{n}, \mathbf{M}, \mathbf{M}^{2}, \ldots, \mathbf{M}^{n-1}\right\}$, where $n$ is chosen to be $N_{t}$. The transmit STM set is given by $\mathcal{S}=\mathcal{D} \times \mathcal{A}$, as earlier.

Remark 1: In contrast to the transcendental extension considered in [33], the aforementioned extension is algebraic. Note that since $M$ is a power of two, $x^{n}+a_{0}$ is irreducible for any $n=2^{l}$, hence the proposed scheme achieves the full transmit diversity order of $n=N_{t}$. For more details on the achievable diversity order, please refer to the proof of Proposition 1 [33].

An important advantage of the proposed AFE-DSM scheme is that it does not suffer from the UDCS issue, which is discussed below.

Proposition 2: In the AFE-DSM scheme, the transmit STM set is closed under multiplication, i.e. $\mathcal{S} \times \mathcal{S} \subseteq \mathcal{S}$.

Proof: Please refer to Appendix C.

Corollary 1: In the AFE-DSM scheme, the differential input constellation size does not grow with the number of transmission blocks $i$.

Proof: The proof directly follows by repeatedly applying Proposition 2.

In the next part of the paper, we extend AFE-DSM to AFEDSM-DR scheme, which trades off diversity order against the throughput, whilst avoiding the UDCS issue.

2) $A F E-D S M-D R$ : In contrast to the FE-DSM-DR, where both the field extensions $K=F(\alpha)$ and $L=K(\beta)$ are transcendental, we consider algebraic extensions in case of AFE-DSM-DR. Specifically, the elements $u_{1}$ and $u_{2}$ in $p_{1}(x)=x^{g}-e^{j u_{1}}$ and $p_{2}(x)=x^{h}-e^{j u_{2}}$ are chosen for ensuring that the associated field extensions are algebraic. Recall that in AFE-DSM $x^{n}-\omega_{M}$ is irreducible, when both $n$ and $M$ are powers of two. Thus, we can choose $u_{1}=2 \pi / M$ and $g$ to be a power of two, which renders the polynomial $p_{1}(x)$ irreducible over $\mathbb{Q}(S)$, hence guaranteeing a transmit diversity order of $g$. Note that in case of AFE-DSM-DR, we have $\mathbf{M}^{g}=e^{j 2 \pi / M} \mathbf{I}_{g}$, which is an important property that assists us in alleviating the UDCS issue. Furthermore, let us consider $p_{2}(x)=x^{h}-\omega_{M}$, where we have opted for $u_{2}=2 \pi / M$. The transmit STM set is given by $\mathcal{S}=\mathcal{D} \times \mathcal{A}$, where $\mathcal{D}$ and $\mathcal{A}$ are of the form (8) and (9), respectively. The following proposition shows that the AFE-DSM-DR advocate does not suffer from the UDCS issue.

Proposition 3: In AFE-DSM-DR scheme, the transmit $\mathrm{STM}$ set is closed under multiplication, i.e. $\mathcal{S} \times \mathcal{S} \subseteq \mathcal{S}$.

Proof: Please refer to Appendix D.

Corollary 2: In the AFE-DSM-DR scheme, the differential input constellation size does not grow with the number of transmission blocks $i$.

Proof: The proof directly follows by repeatedly applying Proposition 3.
Thus, from Corollary 1 and Corollary 2 it becomes evident that the proposed AFE-DSM and AFE-DSM-DR schemes do not suffer from the UDCS issue. In the next part of the paper, we study the AFE-DSM and AFE-DSM-DR schemes in conjunction with differential ASK constellations.

\section{Proposed APSK Aided AFE-DSM AND AFE-DSM-DR}

So far we have considered only PSK constellation while studying the AFE-DSM and AFE-DSM-DR schemes. In this section we employ ASK in conjunction with the PSK (APSK) in order to further improve the bandwidth efficiency of the proposed AFE-DSM and AFE-DSM-DR schemes. Furthermore, we conceive several novel detection schemes for striking a performance complexity trade-off.

Let $L_{a}$ denote the number of discrete amplitude levels, which is assumed to be a power of two. Let these amplitude levels be given by $\left\{1, \alpha, \alpha^{2}, \ldots, \alpha^{L_{a}-1}\right\}$, where $\alpha>1$. The $\left(L_{a} M\right)$-APSK signal set is then given by $\left\{\alpha^{k} \omega_{M}^{l}\right\}_{0 \leq k \leq L_{a}-1,0 \leq l \leq M-1}$. In order to have a unity average power, the signal constellation is assumed to be normalized by $\sqrt{\beta}$, where $\beta=\frac{\sum_{i=0}^{L_{a}-1} \alpha^{2 i}}{L_{a}}$. The encoding of the transmission amplitude in the $i^{\text {th }}$ transmission block is given by $\gamma_{i}=\alpha^{k_{i}} / \gamma_{i-1}$, where $0 \leq k_{i} \leq L_{a}-1$ is chosen based on the $\log _{2}\left(L_{a}\right)$ bits belonging to the $i^{\text {th }}$ transmitted data block.

\section{A. Proposed APSK aided AFE-DSM and AFE-DSM-DR}

Recall from Section III-A1 that the set $\mathcal{D}$ consists of scaled identity matrices of the form $s \mathbf{I}_{n}$, where $s \in L$ PSK signal set. In contrast to this, while employing APSK constellations, we have $\mathcal{D}=\left\{s \mathbf{I}_{n} \mid s \in\left(L_{a} L\right)\right.$-APSK $\}$, where the $|s|=\gamma_{i}=\alpha^{k_{i}} / \gamma_{i-1}$ is decided based on $\log _{2}\left(L_{a}\right)$ bits belonging to the $i^{\text {th }}$ transmission data block. Furthermore, proceeding along the lines of Proposition 2 and Corollary 1, it can be readily seen that employing APSK constellations does not lead to any UDCS issues in AFE-DSM, i.e. we have finitecardinality differential input constellation. Similarly, in case of AFE-DSM-DR we have

$\mathcal{D}=\left\{\operatorname{diag}\left(s_{1} \mathbf{A}_{1}, s_{2} \mathbf{A}_{2}, \ldots, s_{h} \mathbf{A}_{h}\right) \mid s_{j} \in L-\mathrm{PSK}, \mathbf{A}_{j} \in \mathcal{A}^{\prime}\right\}$,

so that the amplitudes of all the PSK symbols $s_{j}$ are differentially encoded as $\left|s_{j}\right|=\gamma_{i}=\alpha^{k_{i}} / \gamma_{i-1}$.

Note that the differential detector of (2) is no longer optimal, when employing APSK constellations and hence better detectors have to be conceived. In the following subsections, we propose the

- Buffered MMSE (B-MMSE) based differential detector;

- Buffered ML (B-ML) differential detector;

which are shown to perform significantly better than the conventional differential detector (CDD) of (2) in Section V.

\section{B. Proposed B-MMSE Detector}

Since employing an APSK signal set renders the transmit matrices of both AFE-DSM and AFE-DSM-DR to be nonunitary, the CDD (2) is no longer optimal. An MMSE based 
differential detector was proposed in [50], which was conceived for overcoming the shortcomings of the CDD in differential transmission schemes employing non-unitary transmit matrices. In this section, we further generalize the MMSE based detector of [50] by considering multiple successive receive matrices instead of two [50] and propose a buffered MMSE based differential detector.

Considering $k+1$ successive receive matrices, we have (12) (given in the next page) where we have assumed that the channel envelope remains constant for $k+1$ successive transmission blocks, i.e. $\left\{\mathbf{H}_{j}\right\}_{j=i-k}^{i}$ are identical. Assuming that $\mathbf{H}_{i}, \sqrt{\rho}$ and $\left\{\mathbf{X}_{j}\right\}_{j=i-k}^{i}$ are known, we have

$$
f\left(\overline{\mathbf{Y}} \mid \mathbf{H}_{i}, \sqrt{\rho}, \overline{\mathbf{X}}\right) \propto \exp \left(-\sum_{j=i-k}^{i}\left\|\mathbf{Y}_{j}-\sqrt{\rho} \mathbf{H}_{i} \mathbf{X}_{j}\right\|_{F}^{2}\right) \text {. }
$$

Let $\Upsilon=\sum_{j=i-k}^{i}\left\|\mathbf{Y}_{j}-\sqrt{\rho} \mathbf{H}_{i} \mathbf{X}_{j}\right\|_{F}^{2}$, which can be equivalently written as

$$
\begin{aligned}
\Upsilon & =\sum_{j=i-k}^{i}\left\|\mathbf{Y}_{j}-\sqrt{\rho} \mathbf{H}_{i} \mathbf{X}_{i-k} \overline{\mathbf{S}}_{j}^{i-k+1}\right\|_{F}^{2}, \\
& \equiv \sum_{j=i-k}^{i}\left\|\mathbf{Y}_{j}-\mathbf{Z} \overline{\mathbf{S}}_{j}^{i-k+1}\right\|_{F}^{2}
\end{aligned}
$$

where $\overline{\mathbf{S}}_{i}^{j}=\mathbf{S}_{j} \mathbf{S}_{j+1} \cdots \mathbf{S}_{i-1} \mathbf{S}_{i}$ and $\overline{\mathbf{S}}_{i}^{j}=\mathbf{I}$, when $i<j$ and $\mathbf{Z}=\sqrt{\rho} \mathbf{H}_{i} \mathbf{X}_{i-k}$. The ML metric in (13) can be minimized w.r.t. $\mathbf{Z}=\sqrt{\rho} \mathbf{H}_{i} \mathbf{X}_{i-k}$ by differentiating $\Upsilon$ w.r.t. $\mathbf{Z}$ and equating it to zero. The following proposition gives the value of $\frac{\partial \Upsilon}{\partial \mathbf{Z}}$.

Proposition 4: Let $\Upsilon$ and $\mathbf{Z}$ be defined as above. Then, we have $\frac{\partial \Upsilon}{\partial \mathbf{Z}}=\mathbf{Z}\left[\sum_{l=0}^{k} \overline{\mathbf{S}}_{i-l}^{i-k+1} \overline{\mathbf{S}}_{i-l}^{i-k+1^{H}}\right]-$ $\left[\sum_{l=0}^{k} \mathbf{Y}_{i-l} \overline{\mathbf{S}}_{i-l}^{i-k+1^{H}}\right]$.

Proof: Please refer to Appendix E.

Now, the specific $\hat{\mathbf{Z}}$ that minimizes (13) is obtained by equating $\frac{\partial \Upsilon}{\partial \mathbf{Z}}$ to zero and solving it for $\mathbf{Z}$, yielding $\frac{\partial \Upsilon}{\partial \mathbf{Z}}=$ $0, \Longrightarrow \hat{\mathbf{Z}} \sum_{l=0}^{k} \overline{\mathbf{S}}_{i-l}^{i-k+1} \overline{\mathbf{S}}_{i-l}^{i-k+1^{H}}-\sum_{l=0}^{k} \mathbf{Y}_{i-l} \overline{\mathbf{S}}_{i-l}^{i-k+1^{H}}=0$, $\Longrightarrow \hat{\mathbf{Z}}=\left(\sum_{l=0}^{k} \mathbf{Y}_{i-l} \overline{\mathbf{S}}_{i-l}^{i-k+1^{H}}\right)\left(\sum_{l=0}^{k} \overline{\mathbf{S}}_{i-l}^{i-k+1} \overline{\mathbf{S}}_{i-l}^{i-k+1^{H}}\right)^{-1}$.

Note that $\hat{\mathbf{Z}}$ is a function of $\mathbf{S}_{i-k}, \mathbf{S}_{i-k+1}, \cdots, \mathbf{S}_{i-1}, \mathbf{S}_{i}$. By using $\hat{\mathbf{S}}_{i-k}, \hat{\mathbf{S}}_{i-k+1}, \cdots, \hat{\mathbf{S}}_{i-1}$, which are the estimates of $\mathbf{S}_{i-k}, \mathbf{S}_{i-k+1}, \cdots, \mathbf{S}_{i-1}$ available from the detector invoked during earlier channel uses, $\hat{\mathbf{Z}}$ can be viewed as being a function of $\mathbf{S}_{i}$ alone. For clarity of presentation, we use $\hat{\mathbf{Z}}\left(\mathbf{S}_{i}\right)$ in order to represent $\hat{\mathbf{Z}}$. Substituting $\hat{\mathbf{Z}}\left(\mathbf{S}_{i}\right)$ into (15), we have $\Upsilon\left(\mathbf{S}_{i}\right)=\sum_{j=i-k}^{i}\left\|\mathbf{Y}_{j}-\hat{\mathbf{Z}}\left(\mathbf{S}_{i}\right) \overline{\mathbf{S}}_{j}^{i-k+1}\right\|_{F}^{2}$.

The proposed B-MMSE detector is given by

$$
\hat{\mathbf{S}}_{i}=\arg \min _{\mathbf{S}_{i} \in \mathcal{S}} \sum_{j=i-k}^{i}\left\|\mathbf{Y}_{j}-\hat{\mathbf{Z}}\left(\mathbf{S}_{i}\right) \overline{\mathbf{S}}_{j}^{i-k+1}\right\|_{F}^{2}
$$

where $\overline{\mathbf{S}}_{i}^{j}=\hat{\mathbf{S}}_{j} \hat{\mathbf{S}}_{j+1} \cdots \hat{\mathbf{S}}_{i-1} \mathbf{S}_{i}$. Note that the proposed BMMSE detector of (16) reduces to that of [50], when $k=1$. We illustrate the proposed B-MMSE detector when $k=2,3$ in the examples provided in the next page.

Remark 2: Although the proposed B-MMSE detector is capable of outperforming the CDD, it will be demonstrated by our simulation results in Section $V$ that it would result in error floors for certain system configurations. The main reason for the emergance of the error-floor is that whenever $\mathbf{S}_{i}$ is erroneously decoded, it affects each of the terms in the summation in (16) during the subsequent decoding, since $\hat{\mathbf{Z}}$ depends on $\hat{\mathbf{S}}_{i-1}, \ldots, \hat{\mathbf{S}}_{i-k}$. Thus, the entire metric is affected by a single instance of erroneous decoding, which in turn causes the errors to propagate. This limitation is overcome by our B-ML detector, which is presented in the next part of the paper.

\section{Proposed B-ML Detector}

Considering the system model of (12) and assuming that the channel $\mathbf{H}_{i}$ is unknown to the receiver, we have

$$
f(\overline{\mathbf{Y}} \mid \rho, \overline{\mathbf{X}}) \propto \frac{\exp \left\{-\operatorname{Tr}\left[\overline{\mathbf{Y}}\left(\mathbf{I}_{(k+1) N_{t}}+\rho \overline{\mathbf{X}}^{H} \overline{\mathbf{X}}\right)^{-1} \overline{\mathbf{Y}}^{H}\right]\right\}}{\operatorname{det}\left(\mathbf{I}_{(k+1) N_{t}}+\rho \overline{\mathbf{X}}^{H} \overline{\mathbf{X}}\right)^{N_{r}}}
$$

By invoking the matrix inversion lemma [52], we have

$$
\left(\mathbf{I}_{(k+1) N_{t}}+\rho \overline{\mathbf{X}}^{H} \overline{\mathbf{X}}\right)^{-1}=\mathbf{I}_{(k+1) N_{t}}-\frac{\overline{\mathbf{X}}^{H} \overline{\mathbf{X}}}{\left(\frac{1}{\rho}+\sum_{l=0}^{k} \gamma_{i-l}^{2}\right)} .
$$

Furthermore, we have

$$
\begin{aligned}
\operatorname{det}\left(\mathbf{I}_{(k+1) N_{t}}+\rho \overline{\mathbf{X}}^{H} \overline{\mathbf{X}}\right)^{N_{r}} & =\operatorname{det}\left(\mathbf{I}_{N_{t}}+\rho \overline{\mathbf{X}} \overline{\mathbf{X}}^{H}\right)^{N_{r}} \\
& =\operatorname{det}\left(\mathbf{I}_{N_{t}}+\rho \sum_{l=0}^{k} \gamma_{i-l}^{2} \mathbf{I}_{N_{t}}\right)^{N_{r}} \\
& =\left(1+\rho \sum_{l=0}^{k} \gamma_{i-l}^{2}\right)^{N_{t} N_{r}}
\end{aligned}
$$

Substituting (18) and (21) in (17), we have

$$
\begin{aligned}
f(\overline{\mathbf{Y}} \mid \rho, \overline{\mathbf{X}}) \propto & \frac{\exp \left\{-\operatorname{Tr}\left[\overline{\mathbf{Y}} \overline{\mathbf{Y}}^{H}-\frac{\overline{\mathbf{Y}} \overline{\mathbf{X}}^{H} \overline{\mathbf{X}} \overline{\mathbf{Y}}^{H}}{\frac{1}{\rho}+\sum_{l=0}^{k} \gamma_{i-l}^{2}}\right]\right\}}{\left(1+\rho \sum_{l=0}^{k} \gamma_{i-l}^{2}\right)^{N_{t} N_{r}}}, \\
& =\frac{\exp \left\{-\|\overline{\mathbf{Y}}\|_{F}^{2}+\frac{\left\|\overline{\mathbf{Y}} \overline{\mathbf{X}}^{H}\right\|_{F}^{2}}{\frac{1}{\rho}+\sum_{l=0}^{k} \gamma_{i-l}^{2}}\right\}}{\left(1+\rho \sum_{l=0}^{k} \gamma_{i-l}^{2}\right)^{N_{t} N_{r}}}, \\
& =\frac{\exp \left\{-\sum_{l=0}^{k}\left\|\mathbf{Y}_{i-l}\right\|_{F}^{2}+\frac{\left\|\sum_{l=0}^{k} \mathbf{Y}_{i-l} \mathbf{x}_{i-l}^{H}\right\|_{F}^{2}}{\frac{1}{\rho}+\sum_{l=0}^{k} \gamma_{i-l}^{2}}\right\}}{\left(1+\rho \sum_{l=0}^{k} \gamma_{i-l}^{2}\right)^{N_{t} N_{r}}} .
\end{aligned}
$$

Taking natural logarithm on either side of (24), we have $\ln f(\overline{\mathbf{Y}} \mid \rho, \overline{\mathbf{X}}) \propto-\sum_{l=0}^{k}\left\|\mathbf{Y}_{i-l}\right\|_{F}^{2}+\frac{\left\|\sum_{l=0}^{k} \mathbf{Y}_{i-l} \mathbf{X}_{i-l}^{H}\right\|_{F}^{2}}{\frac{1}{\rho}+\sum_{l=0}^{k} \gamma_{i-l}^{2}}-$ $N_{t} N_{r} \ln \left(1+\rho \sum_{l=0}^{k} \gamma_{i-l}^{2}\right)$.

The proposed B-ML detector is given by $\hat{\mathbf{S}}_{i}=$ $\arg \min _{\mathbf{S}_{i} \in \mathcal{S}} \Psi\left(\mathbf{S}_{i}\right)$, where

$$
\begin{array}{r}
\Psi\left(\mathbf{S}_{i}\right)=-\sum_{l=0}^{k}\left\|\mathbf{Y}_{i-l}\right\|_{F}^{2}+\frac{\left\|\sum_{l=0}^{k} \mathbf{Y}_{i-l} \hat{\mathbf{X}}_{i-l}^{H}\right\|_{F}^{2}}{\frac{1}{\rho}+\gamma_{i}^{2}+\sum_{l=1}^{k} \hat{\gamma}_{i-l}^{2}} \\
-N_{t} N_{r} \ln \left(1+\rho \gamma_{i}^{2}+\rho \sum_{l=1}^{k} \hat{\gamma}_{i-l}^{2}\right),
\end{array}
$$

such that $\hat{\mathbf{X}}_{i}=\hat{\mathbf{X}}_{i-1} \mathbf{S}_{i}$ and $\left\{\hat{\mathbf{X}}_{i-j}\right\}_{j=1}^{k}$ are the estimates 


$$
\underbrace{\left[\mathbf{Y}_{i}, \mathbf{Y}_{i-1}, \ldots, \mathbf{Y}_{i-k}\right]}_{\overline{\mathbf{Y}}}=\sqrt{\rho \mathbf{H}_{i}} \underbrace{\left[\mathbf{X}_{i}, \mathbf{X}_{i-1}, \ldots, \mathbf{X}_{i-k}\right]}_{\overline{\mathbf{X}}}+\left[\mathbf{N}_{i}, \mathbf{N}_{i-1}, \ldots, \mathbf{N}_{i-k}\right]
$$

Example 3: The B-MMSE metric associated with $k=2$ is given as follows:

$$
\Upsilon\left(\mathbf{S}_{i}\right)=\left\|\mathbf{Y}_{i}-\hat{\mathbf{Z}}\left(\mathbf{S}_{i}\right) \hat{\mathbf{S}}_{i-1} \mathbf{S}_{i}\right\|_{F}^{2}+\left\|\mathbf{Y}_{i-1}-\hat{\mathbf{Z}}\left(\mathbf{S}_{i}\right) \hat{\mathbf{S}}_{i-1}\right\|_{F}^{2}+\left\|\mathbf{Y}_{i-2}-\hat{\mathbf{Z}}\left(\mathbf{S}_{i}\right)\right\|_{F}^{2},
$$

where

$$
\hat{\mathbf{Z}}\left(\mathbf{S}_{i}\right)=\left[\mathbf{Y}_{i}\left(\mathbf{S}_{i}^{H} \hat{\mathbf{S}}_{i-1}^{H}\right)+\mathbf{Y}_{i-1} \hat{\mathbf{S}}_{i-1}^{H}+\mathbf{Y}_{i-2}\right]\left[\hat{\mathbf{S}}_{i-1}\left(\mathbf{S}_{i} \mathbf{S}_{i}^{H}\right) \hat{\mathbf{S}}_{i-1}^{H}+\hat{\mathbf{S}}_{i-1} \hat{\mathbf{S}}_{i-1}^{H}+\mathbf{I}\right]^{-1}
$$

Example 4: The B-MMSE metric associated with $k=3$ is given as follows:

$$
\Upsilon\left(\mathbf{S}_{i}\right)=\left\|\mathbf{Y}_{i}-\hat{\mathbf{Z}}\left(\mathbf{S}_{i}\right) \hat{\mathbf{S}}_{i-2} \hat{\mathbf{S}}_{i-1} \mathbf{S}_{i}\right\|_{F}^{2}+\left\|\mathbf{Y}_{i-1}-\hat{\mathbf{Z}}\left(\mathbf{S}_{i}\right) \hat{\mathbf{S}}_{i-2} \hat{\mathbf{S}}_{i-1}\right\|_{F}^{2}+\left\|\mathbf{Y}_{i-2}-\hat{\mathbf{Z}}\left(\mathbf{S}_{i}\right) \hat{\mathbf{S}}_{i-2}\right\|_{F}^{2}+\left\|\mathbf{Y}_{i-3}-\hat{\mathbf{Z}}\left(\mathbf{S}_{i}\right)\right\|_{F}^{2},
$$

where

$\hat{\mathbf{Z}}\left(\mathbf{S}_{i}\right)=\left[\mathbf{Y}_{i} \mathbf{S}_{i}^{H} \hat{\mathbf{S}}_{i-1}^{H} \hat{\mathbf{S}}_{i-2}^{H}+\mathbf{Y}_{i-1} \hat{\mathbf{S}}_{i-1}^{H} \hat{\mathbf{S}}_{i-2}^{H}+\mathbf{Y}_{i-2} \hat{\mathbf{S}}_{i-2}^{H}+\mathbf{Y}_{i-3}\right]\left[\hat{\mathbf{S}}_{i-2} \hat{\mathbf{S}}_{i-1} \mathbf{S}_{i} \mathbf{S}_{i}^{H} \hat{\mathbf{S}}_{i-1}^{H} \hat{\mathbf{S}}_{i-2}^{H}+\hat{\mathbf{S}}_{i-2} \hat{\mathbf{S}}_{i-1} \hat{\mathbf{S}}_{i-1}^{H} \hat{\mathbf{S}}_{i-2}^{H}+\hat{\mathbf{S}}_{i-2} \hat{\mathbf{S}}_{i-2}^{H}+\mathbf{I}\right]^{-1}$.

based on the previously detected transmit matrices. Similarly, $\left\{\hat{\gamma}_{j}\right\}_{j=1}^{k}$ are the estimates of the transmit amplitude of the previous $k$ transmit matrices.

\section{Simulation Results and Discussions}

Simulation parameters: In calculating a bit error rate (BER) of $10^{-m}$ we have used at least $10^{m+2}$ bits. Each BER point is averaged over 10,000 channel realizations. In all our simulations, we have used block Rayleigh fading channels with a coherence duration of 1000 channel uses. The AFE-DSM and AFE-DSM-DR schemes are assumed to use $u_{1}=2 \pi / M$ and $\left(u_{1}, u_{2}\right)=(2 \pi / M, 2 \pi / M)$, while employing an $M$-PSK signal set, respectively. In case of the AFE-DSMDR scheme, we have considered $g=h=2$ and $N_{t}=4$. The same set of parameters are used in case of its APSK aided counterparts as well. In the simulation results, the parameter $k$ denotes the buffer length of the B-MMSE and B-ML detectors and the APSK signal set is assumed to have $L_{a}=2$ and $\alpha=2$.

Let us first compare the BER performance of the existing FE-DSM-DR scheme to that of the proposed AFE-DSMDR scheme ${ }^{4}$. Fig. 2 compares the BER performance of the existing FE-DSM-DR to that of the AFE-DSM-DR scheme employing various PSK constellations ranging from BPSK to 16-PSK, where both the schemes are assumed to employ the conventional differential detector at the receiver. It is evident from Fig. 2 that the proposed AFE-DSM-DR scheme achieves nearly the same performance as that of the FE-DSM scheme, i.e. it does not suffer from any performance loss. Thus the proposed AFE-DSM and AFE-DSM-DR schemes provide the maximum achievable diversity advantage, while avoiding the UDCS issue.

Fig. 3 portrays the BER performance of the B-MMSE detector recorded for various values of buffer length $k$, when employing the APSK aided AFE-DSM scheme using $N_{t}=N_{r}=2$. Specifically, Fig. 3(a) corresponds to the

\footnotetext{
${ }^{4}$ Note that the optimal phase angle $\left(e^{j u_{1}}\right)$ in case of FE-DSM and AFEDSM schemes are same and hence their BER performances are identical.
}

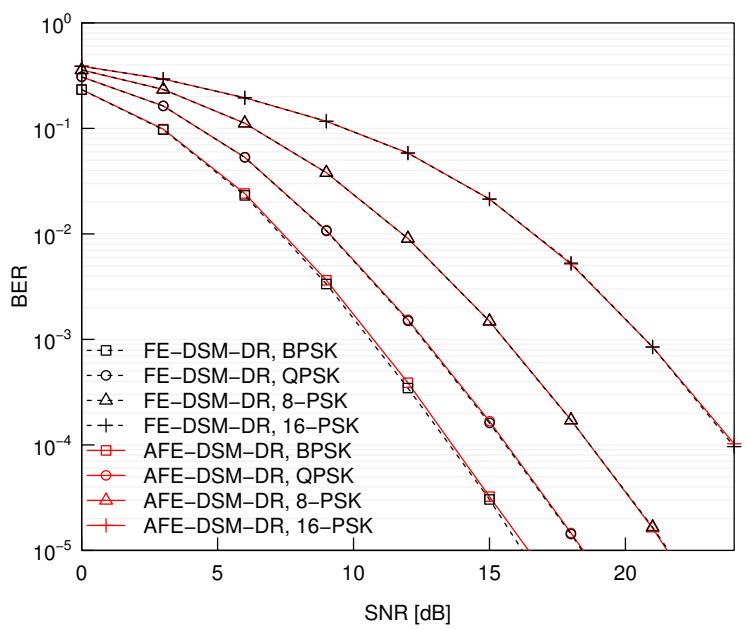

Fig. 2. Comparison of the BER performance of FE-DSM-DR with that of AFE-DSM-DR scheme in a system having $N_{t}=4, N_{r}=2$, and employing various PSK signal sets.

throughput of $2 \mathrm{bpcu}$, while Fig. 3(b) corresponds to $3 \mathrm{bpcu}$. It is evident from Fig. 3 that for larger values of $k$ the BMMSE detector suffers from an error floor. Fig. 4 portrays the BER performance of the B-ML detector in the aforementioned system. It can be seen from the figure that unlike the BMMSE detector, the B-ML detector does not suffer from any error floor. From Fig. 3 and Fig. 4, we can also infer that the performance gain of both detectors diminishes as $k$ increases. Furthermore, we can also see from Fig. 3 and Fig. 4 that the performance gain attained drops as the throughput increases.

Fig. 5 portrays the BER performance of the B-ML detector for various values of buffer length $k$, when employing the APSK aided AFE-DSM-DR scheme using $N_{t}=4$ and $N_{r}=$ 2. Similarly to the AFE-DSM scheme, it is evident that the performance gain erodes as $k$ increases. This can be attributed to the nature of differential encoding, where any transmit STM is encoded based on the product of the DMs corresponding 

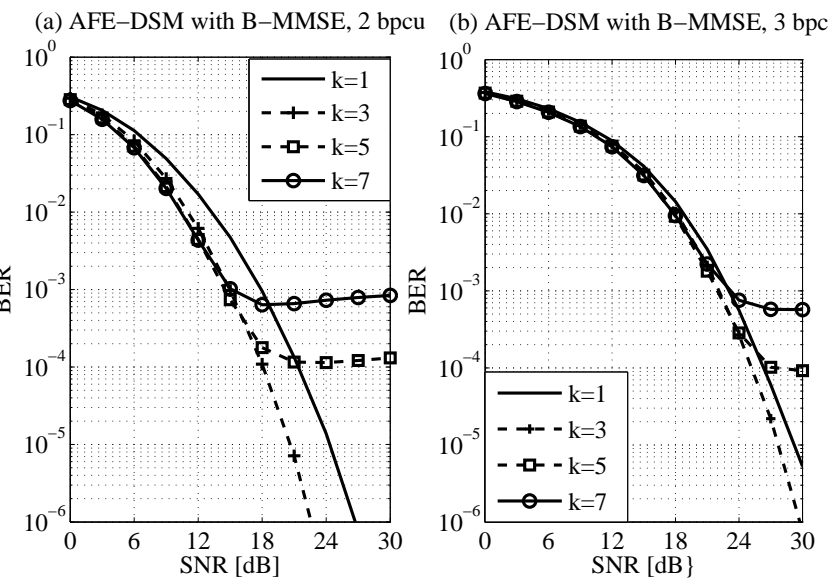

Fig. 3. Comparison of the BER performance by B-MMSE detector for various values of buffer length $k$. Plot (a) and Plot (b) correspond to the APSK aided AFE-DSM scheme operating at 2 bpcu and 3 bpcu, respectively.
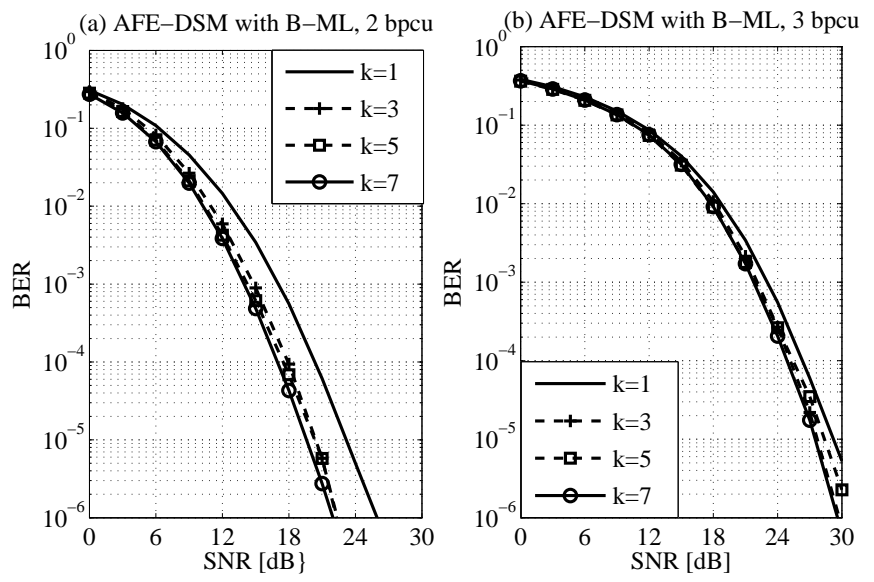

Fig. 4. Comparison of the BER performance by B-ML detector for various values of buffer length $k$. Plot (a) and Plot (b) correspond to the APSK aided AFE-DSM scheme operating at 2 bpcu and 3 bpcu, respectively.

to the current and the previous channel uses. ${ }^{5}$ Note that in Fig. 5(b), the BER performance of $k=3$ is slightly better than that of $k=7$. This is attributed to the smaller buffer length, which makes the system more resilient to error propagation. It is important to note that longer buffers do not necessarily imply better performance, since longer buffers are more prone to error propagation. Let us now compare the performance of the proposed detectors against that of the existing CDD and its coherent counterpart.

Fig. 6 and Fig. 7 compare the BER performance of the proposed detectors against that of the CDD and its coherent counterpart, when employing the APSK aided AFEDSM scheme associated with $N_{t}=N_{r}=2$. In case of coherent detection, the receiver is assumed to have perfect CSI and employ the ML detector. Specifically, Fig. 6 and Fig. 7 correspond to the throughput of $2 \mathrm{bpcu}$ and $3 \mathrm{bpcu}$,

\footnotetext{
${ }^{5}$ Note that owing to the severe error floors exhibited by the B-MMSE detector in case of AFE-DSM-DR scheme, we restrict our further studies to only B-ML when employing AFE-DSM-DR.
}

(a) AFE-DSM-DR with B-ML,

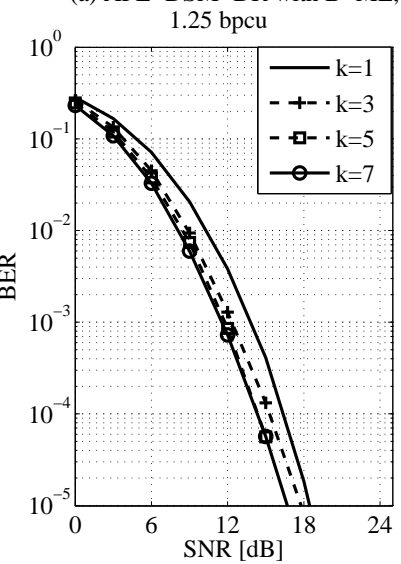

(b) AFE-DSM-DR with B-ML,

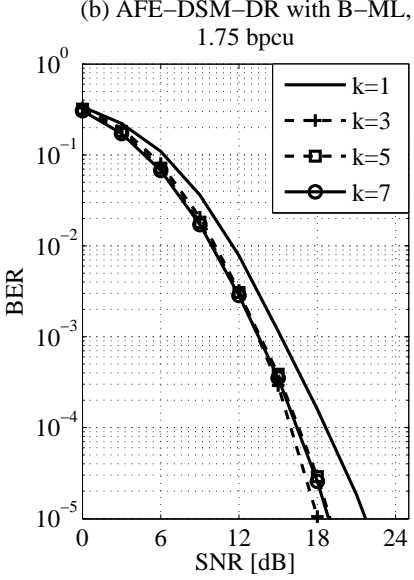

Fig. 5. Comparison of the BER performance by B-ML detector for various values of buffer length $k$. Plot (a) and Plot (b) correspond to the APSK aided AFE-DSM-DR scheme operating at $1.25 \mathrm{bpcu}$ and $1.75 \mathrm{bpcu}$, respectively.
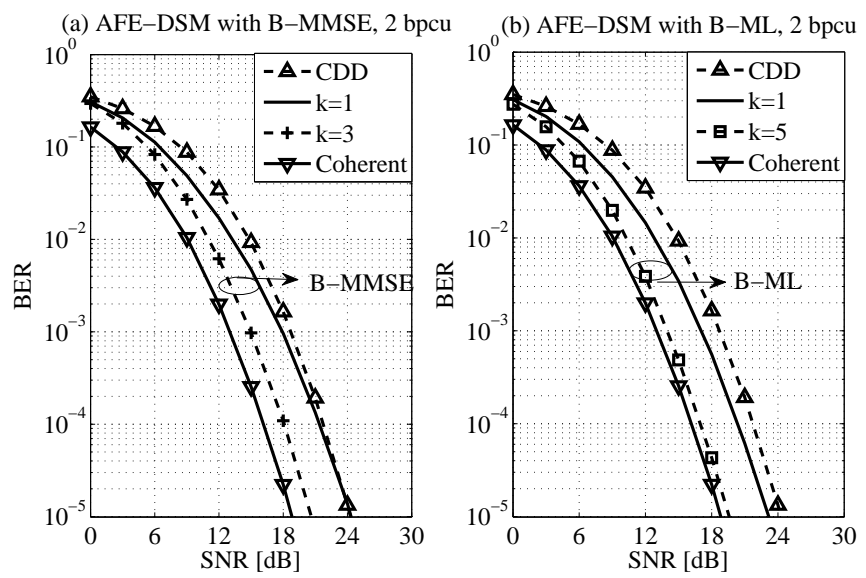

Fig. 6. Comparison of the BER performance by the proposed B-MMSE and B-ML detectors with that of the existing CDD and the coherent counterpart, when employing APSK aided AFE-DSM scheme operating at 2 bpcu. Plot (a) and Plot (b) correspond to the B-MMSE and B-ML detectors, respectively.

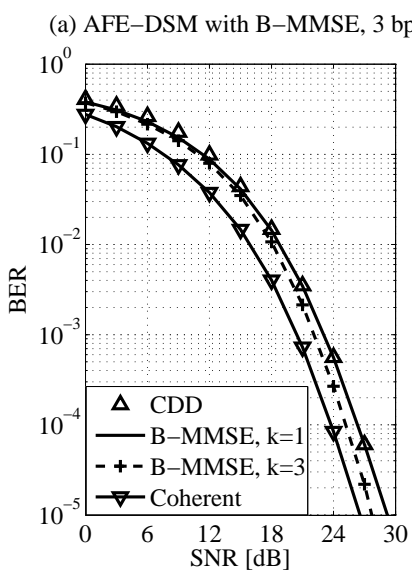

(b) AFE-DSM with B-ML, 3 bpcu

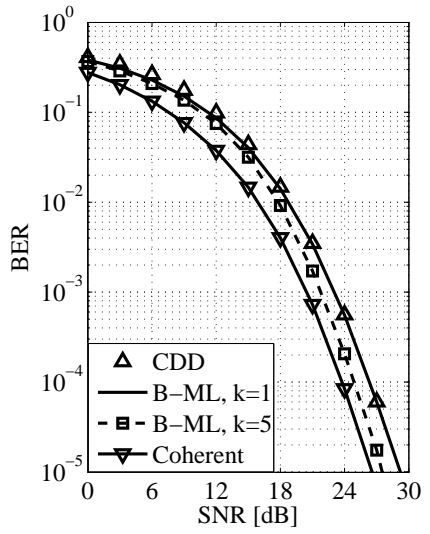

Fig. 7. Comparison of the BER performance by the proposed B-MMSE and B-ML detectors with that of the existing CDD and the coherent counterpart, when employing APSK aided AFE-DSM scheme operating at 3 bpcu. Plot (a) and Plot (b) correspond to the B-MMSE and B-ML detectors, respectively. 

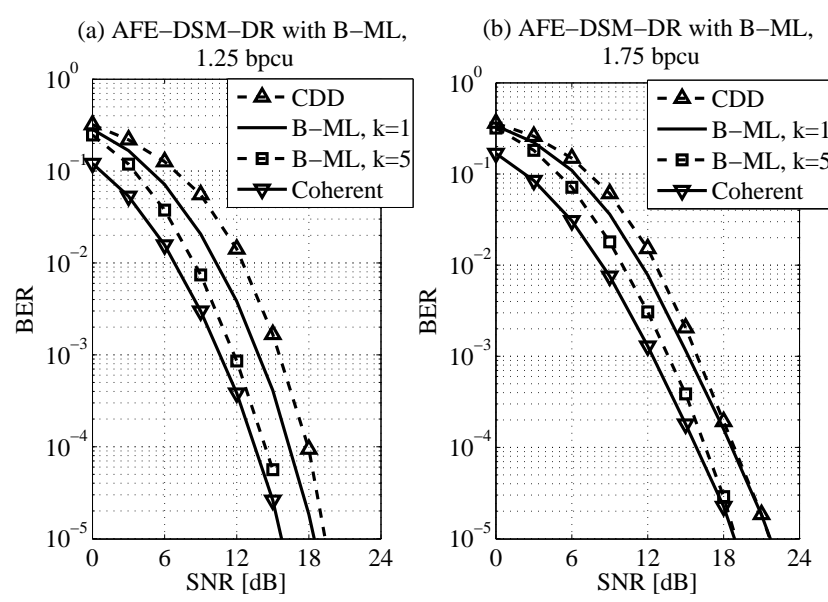

Fig. 8. Comparison of the BER performance by the proposed B-ML detector with that of the existing CDD and the coherent counterpart, when employing APSK aided AFE-DSM-DR scheme. Plot (a) and Plot (b) correspond to the throughputs of $1.25 \mathrm{bpcu}$ and $1.75 \mathrm{bpcu}$, respectively.

respectively. It is evident from both Figures 6 and 7 that the proposed detectors are capable of outperforming the CDD, while attaining a performance close to that of their coherent counterparts. Specifically, we observe from Fig. 6(a) that the B-MMSE detector $(k=3)$ attains about $3 \mathrm{~dB}$ performance gain over the CDD and hence is only about $1 \mathrm{~dB}$ away from that of its coherent counterpart at a BER of $10^{-5}$. We observe from Fig. 6(b) that at a BER of $10^{-5}$ the performance of the B-ML detector $(k=5)$ is only about $0.5 \mathrm{~dB}$ away from that of its coherent counterpart. Similar observations can be drawn from Fig. 7 as well.

Fig. 8 compares the BER performance of the B-ML detector when employing the APSK aided AFE-DSM-DR scheme associated with $N_{t}=4$ and $N_{r}=2$. It can be observed from Fig. 8 that the performance of the B-ML detector $(k=5)$ nearly coincides with that of its coherent counterpart, thus bridging the performance gap between the CDD and the coherent detector. Note that the differential encoding makes the successive transmit STMs dependent on previous ones. This fact is exploited by the B-ML detector, which makes use of the previously detected STMs in order to improve the detection performance. Let us now study the computational complexity imposed by the various detectors. The CDD in (2) would require $2|\mathcal{S}| N_{r} N_{t}$ number of complex-valued multiplications. The B-MMSE detector in (16) would impose $(2 k+1) N_{r} N_{t}|\mathcal{S}|$ number of complex-valued multiplications for computing $\Upsilon\left(\mathbf{S}_{i}\right)$ and about $\left(k^{2} N_{t}+k N_{t} N_{r}+(k-1) N_{t}\right)|\mathcal{S}|$ number of complexvalued multiplications for computing $\hat{\mathbf{Z}}\left(\mathbf{S}_{i}\right)$. Furthermore, the B-ML detector in (25) would require $2 N_{r} N_{t}(k+1)+N_{r} N_{t}|\mathcal{S}|$ number of complex-valued multiplications for the computation of $\Psi\left(\mathbf{S}_{i}\right)$. Note that the natural logarithm in case of the BML detector in (25) can be implemented using a look-up table. Figure 9 gives the variation of computational complexity as a function of $N_{t}$ and $N_{r}$. Specifically, plot(a) shows the variation of the computational complexity as a function of $N_{t}$, where $N_{r}=2$ and $k=3$ are fixed, whereas plot(b) gives the variation of the computational complexity as a function
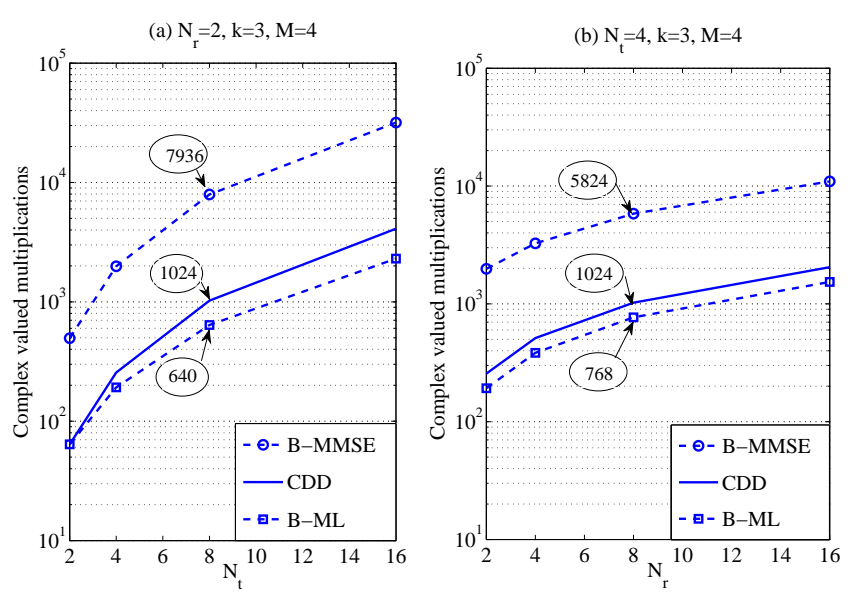

Fig. 9. Comparison of the computational complexity of the proposed detectors with that of the CDD. Plot(a) gives the variation of the computational complexity as a function of $N_{t}$, where $N_{r}=2$ and $k=3$ are fixed, whereas plot(b) gives the variation of the computational complexity as a function of $N_{r}$, where $N_{t}=4$ and $k=3$ are fixed.

of $N_{r}$ where $N_{t}=4$ and $k=3$ are fixed. It is evident from Fig. 9 that the computational complexity of the B-ML detector is much lower than that of the B-MMSE detector and it is comparable to that of CDD. Specifically, when $N_{t}=8$, $N_{r}=2$, and $k=3$, the computational complexity of the B-ML detector is about $91 \%$ lower than that of B-MMSE detector and about $37 \%$ lower than that of CDD. Furthermore, when $N_{t}=4, N_{r}=8$, and $k=3$ the computational complexity of the B-ML detector is about $86 \%$ lower than that of B-MMSE detector and about $25 \%$ lower than that of CDD.

Let us now study the sensitivity of the proposed detectors to channel correlations in time. In order to study the sensitivity of the proposed detectors to channel variations, we consider a rapidly varying channel where each channel block of length $B L$ starts with a channel realization $\mathbf{H}_{1}$ and fades into an independent channel realization $\mathbf{H}_{2}$ in discrete steps of $1 / B L$. That is, the channel matrix in the $i^{\text {th }}$ channel use is given by $\mathbf{H}_{i}=\frac{B L-i}{B L} \mathbf{H}_{1}+\frac{i}{B L} \mathbf{H}_{2}$ for $1 \leq i \leq B L$. Figure 10 compares the variation in the BER in case of the proposed detectors in an APSK aided AFE-DSM system having $N_{t}=N_{r}=2$ and operating at 2 bpcu. Specifically, Fig. 10(a) corresponds to the case where the operating SNR is $18 \mathrm{~dB}$, whereas Fig. 10(b) corresponds to the case of $24 \mathrm{~dB}$. It is evident from Fig. 10 that the proposed detectors retain their advantage at all values of $B L$ in comparison to the existing CDD. Furthermore, at a BER of $10^{-4}$, the proposed B-ML detector is observed to give an SNR gain of $2.5 \mathrm{~dB}$ with respect to the $\mathrm{CDD}$ when $B L=20$ and about $2 \mathrm{~dB}$ when $B L=60$. Thus, the proposed detector not only requires low-computational complexity but also gives beneficial SNR gains.

Let us now study the sensitivity of the proposed detectors to channel correlations in space. We assume the Kronecker channel model for simulating the correlated channel condition, which is characterized by $\mathbf{H}_{\text {cor }}=\mathbf{R}_{r}^{1 / 2} \mathbf{H} \mathbf{R}_{t}^{1 / 2}$, where $\mathbf{R}_{r}$ and $\mathbf{R}_{t}$ are the receive and transmit correlation matrices of 

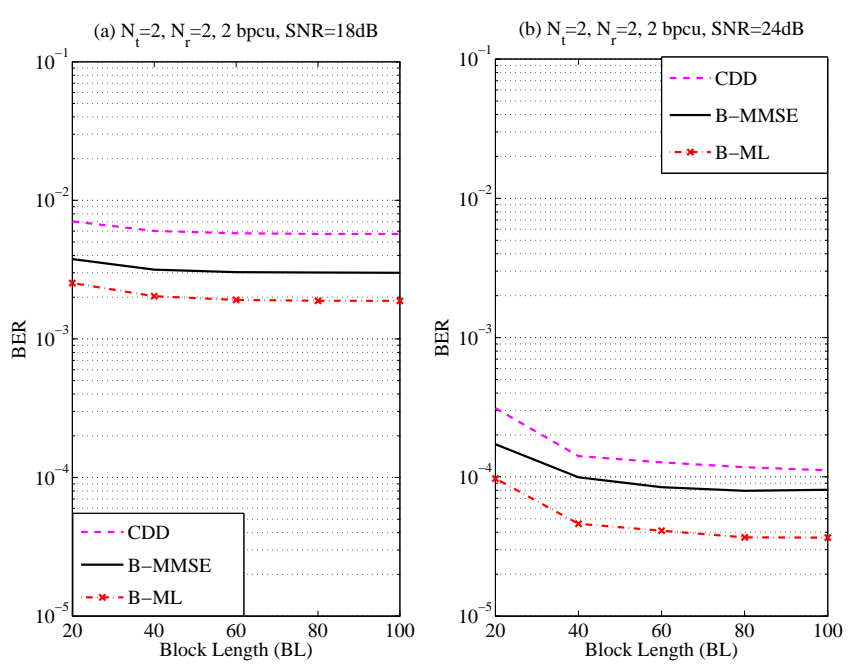

Fig. 10. Variation of the BER as a function of channel block length in an APSK aided AFE-DSM system having $N_{t}=N_{r}=2$ and operating at 2 bpcu. Plot(a) corresponds to the case where the operating SNR is $18 \mathrm{~dB}$, whereas plot(b) corresponds to the case of $24 \mathrm{~dB}$.

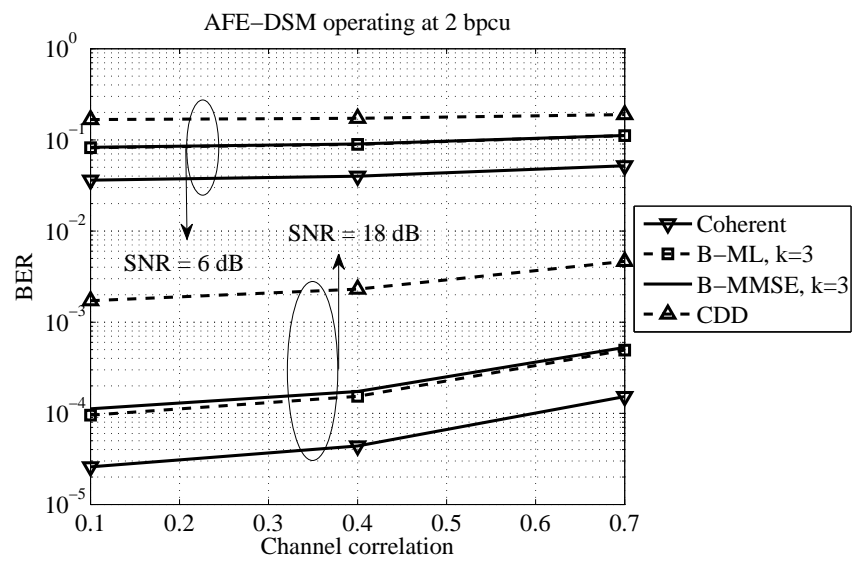

Fig. 11. This figure depicts the sensitivity of the proposed B-MMSE and B-ML detectors to channel correlations in comparison with that of the CDD and the coherent counterpart when employing APSK aided AFE-DSM scheme operating at 2 bpcu.

dimensions $N_{r} \times N_{r}$ and $N_{t} \times N_{t}$, respectively. The $(i, j)^{\text {th }}$ element of these matrices is given by $\sigma^{|i-j|}$, where $0 \leq \sigma \leq 1$ is the correlation coefficient. Fig. 11 and Fig. 12 compare the BER performance of the proposed as well as of the existing detectors in correlated channel conditions. Specifically, Fig. 11 corresponds to the APSK aided AFE-DSM scheme operating at 2 bpcu, whereas Fig. 12 corresponds to the APSK aided AFE-DSM-DR scheme operating at 1.25 bpcu. It is evident from Fig. 11 and Fig. 12 that the proposed detectors are only as sensitive as the CDD or the coherent detector to the channel correlations. It can also be observed that the performance gain of the proposed detectors w.r.t. the CDD remains constant for various channel correlation conditions.

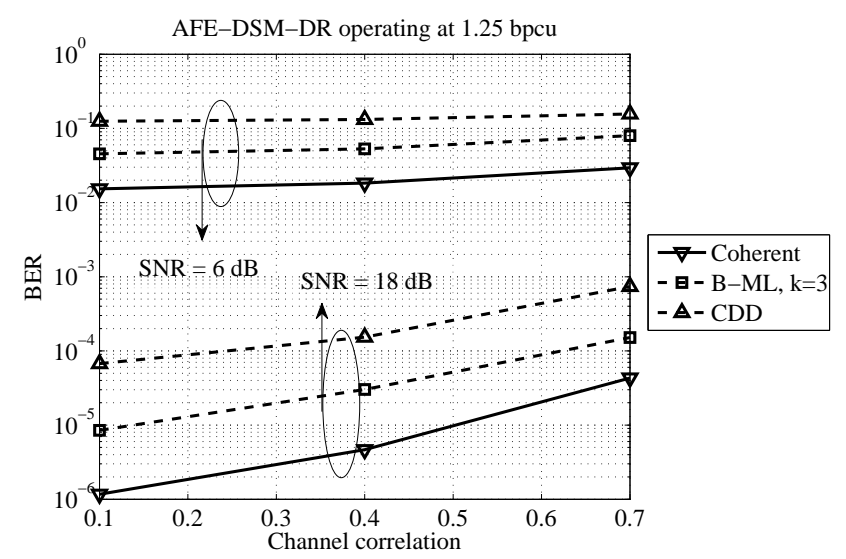

Fig. 12. This figure depicts the sensitivity of the proposed B-ML detector to channel correlations in comparison with that of the CDD and the coherent counterpart when employing APSK aided AFE-DSM-DR scheme operating at $1.25 \mathrm{bpcu}$.

\section{CONClusions}

We have identified a deficiency of differential encoding termed as the UDCS issue and studied the various existing schemes in the light of this phenomenon. Then the AFE-DSM and AFE-DSM-DR schemes were conceived for overcoming the UDCS issue without compromising the attainable diversity advantage. Furthermore, APSK aided schemes were conceived for further exploiting the available degrees of freedom. Additionally, a pair of novel detection schemes were conceived, which outperform the CDD. Our simulation results have demonstrated that the proposed detection schemes are capable of bridging the performance gap between the CDD and the coherent detector, while retaining the same search complexity as that of the CDD or the coherent detector.

\section{APPENDIX A PROOF OF LEMMA 1}

Proof: In order to prove the lemma, it is sufficient to prove that $(i \theta)_{2 \pi}+\frac{2 \pi}{M}\left(\sum_{l=1}^{i} k_{l}\right)_{M} \neq(m \theta)_{2 \pi}+$ $\frac{2 \pi}{M}\left(\sum_{r=1}^{m} k_{r}\right)_{M}$ for $1 \leq m<i$, where $(u)_{t}=u$ modulo $t$. Assuming that

$$
(i \theta)_{2 \pi}+\frac{2 \pi}{M}\left(\sum_{l=1}^{i} k_{l}\right)_{M}=(m \theta)_{2 \pi}+\frac{2 \pi}{M}\left(\sum_{r=1}^{m} k_{r}\right)_{M},
$$

we have $((i-m) \theta)_{2 \pi}=\frac{2 \pi}{M}\left(\sum_{r=1}^{m} k_{r}\right)_{M}-\frac{2 \pi}{M}\left(\sum_{l=1}^{i} k_{l}\right)_{M}=$ $\frac{2 \pi k^{\prime}}{M}$ for some $-(M-1) \leq k^{\prime} \leq(M-1)$. Furthermore, we have $(i-m) \theta=2 \pi q+\frac{2 \pi k^{\prime}}{M}$, which implies that $\frac{M(i-m) \theta}{2\left(q L+k^{\prime}\right)}=\pi$, which contradicts the fact that $\pi$ is transcendental. Thus the equality in (26) does not hold. This concludes the proof.

\section{APPENDIX B}




\section{ProOF OF PROPOSITION 1}

Proof: The differential input constellation at the $i^{\text {th }}$ transmission block is given by

$$
\begin{aligned}
\mathcal{S}_{i} & =\underbrace{\mathcal{S} \times \mathcal{S} \times \cdots \times \mathcal{S}}_{i \text { times }}=(\mathcal{D} \times \mathcal{A}) \times(\mathcal{D} \times \mathcal{A}) \times \cdots \times(\mathcal{D} \times \mathcal{A}) \\
& =\mathcal{D} \times(\mathcal{A} \times \mathcal{A} \times \cdots \times \mathcal{A}) \equiv e^{j \frac{2 \pi}{M} k^{\prime}}\left\{\mathbf{M}^{\sum_{l=1}^{i} q_{l}}\right\}_{0 \leq\left\{q_{l}\right\}_{l=1}^{i} \leq n-1},
\end{aligned}
$$

where $0 \leq k^{\prime} \leq M-1$. Considering the worst case $^{6}$ of $q_{l}=n-1$ for $1 \leq l \leq i$ and letting $i(n-1)=q n+r$, so that $0 \leq r \leq n-1$, we have $\mathcal{S}_{i} \equiv e^{j \frac{2 \pi}{M} k^{\prime}}\left\{\mathbf{M}^{i(n-1)}\right\} \equiv$ $e^{j \frac{2 \pi}{M} k^{\prime}}\left\{\mathbf{M}^{q n+r}\right\} \equiv e^{j \frac{2 \pi}{M} k^{\prime}} e^{j q u_{1}}\left\{\mathbf{M}^{r}\right\}_{0<r<n-1}$, where we have exploited the fact that $\mathbf{M}^{n}=e^{j \bar{u}_{1}} \overline{\mathbf{I}}_{n}$. Since $e^{j u_{1}}$ is transcendental, it follows from Lemma 1 that the set $\mathcal{S}_{i}$ grows by $q M$ symbols over $i$ transmission blocks. This concludes the proof.

\section{ApPEndiX C \\ PROOF OF PROPOSITION 2}

Proof: We have $\mathcal{S} \times \mathcal{S}=(\mathcal{D} \times \mathcal{A}) \times(\mathcal{D} \times \mathcal{A})=\mathcal{D} \times$ $(\mathcal{A} \times \mathcal{A}) \equiv e^{j \frac{2 \pi}{M} k^{\prime}}\left(\mathbf{M}^{q_{1}} \mathbf{M}^{q_{2}}\right)=e^{j \frac{2 \pi}{M} k^{\prime}} \mathbf{M}^{q_{1}+q_{2}}$, where $0 \leq$ $q_{1}, q_{2} \leq(n-1)$ and $0 \leq k^{\prime}<M$. Letting $q_{1}+q_{2}=q n+r$, so that $0 \leq r<M$, we have $\mathcal{S} \times \mathcal{S}=e^{j \frac{2 \pi}{M} k^{\prime}} \mathbf{M}^{q_{1}+q_{2}}=$ $e^{j \frac{2 \pi}{M} k^{\prime}} \mathbf{M}^{q n+r}=e^{j \frac{2 \pi}{M} k^{\prime}} \mathbf{M}^{q n} \mathbf{M}^{r}=e^{j \frac{2 \pi}{M} k^{\prime \prime}} \mathbf{M}^{r} \in \mathcal{S}$, where $k^{\prime \prime}=\left(k^{\prime}+q\right)_{M}$ and we have exploited the fact that $\mathbf{M}^{q n}=$ $e^{j 2 \pi q / M} \mathbf{I}_{n}$. This concludes the proof.

\section{Appendix D}

\section{PROOF OF PROPOSITION 3}

\section{Proof: Considering}

$$
\mathcal{S} \times \mathcal{S}=(\mathcal{D} \times \mathcal{A}) \times(\mathcal{D} \times \mathcal{A})=\mathbf{D}_{1} \mathbf{N}^{\prime q_{1}} \mathbf{D}_{2} \mathbf{N}^{\prime q_{2}},
$$

where $\mathbf{D}_{1}, \mathbf{D}_{2} \in \mathcal{D}(8), \mathbf{N}^{\prime q_{1}}, \mathbf{N}^{\prime q_{2}} \in \mathcal{A}(9)$ and $0 \leq q_{1}, q_{2}<$ $h$. It can be readily seen that $\mathbf{N}^{\prime q_{1}} \mathbf{D}_{2}=\mathbf{D}_{2}^{\left(q_{1}\right)} \mathbf{N}^{\prime q_{1}}$, where $\mathbf{D}_{2}^{\left(q_{1}\right)} \in \mathcal{D}$ is a block diagonal matrix, whose elements are the same as those of $\mathbf{D}_{2}$, except that they are circularly shifted by $q_{1}$ blocks down along the diagonal. Thus, from (29) we have $\mathcal{S} \times \mathcal{S}=\mathbf{D}_{1} \mathbf{D}_{2}^{\left(q_{1}\right)} \mathbf{N}^{\prime q_{1}} \mathbf{N}^{\prime q_{2}}=\mathbf{D}^{\prime} \mathbf{N}^{\prime q_{1}+q_{2}}=$ $\mathbf{D}^{\prime}\left(\mathbf{N} \otimes \mathbf{I}_{g}\right)^{q_{1}+q_{2}}=\mathbf{D}^{\prime}\left(\mathbf{N}^{q_{1}+q_{2}} \otimes \mathbf{I}_{g}\right)$. Letting $q_{1}+q_{2}=q h+$ $r$ such that $0 \leq r<h$, we have $\mathcal{S} \times \mathcal{S}=\mathbf{D}^{\prime}\left(\mathbf{N}^{q h+r} \otimes \mathbf{I}_{g}\right)=$ $\mathbf{D}^{\prime}\left(\mathbf{N}^{q h} \mathbf{N}^{r} \otimes \mathbf{I}_{g}\right)=\mathbf{D}^{\prime} e^{j 2 \pi q / M}\left(\mathbf{N}^{r} \otimes \mathbf{I}_{g}\right) \in \mathcal{S}$, where we have exploited the fact that $\mathbf{N}^{q h}=e^{j 2 \pi q / M} \mathbf{I}_{h}, \mathbf{D}^{\prime} e^{j 2 \pi q / M} \in$ $\mathcal{D}$ and $\left(\mathbf{N}^{r} \otimes \mathbf{I}_{g}\right) \in \mathcal{A}$. This concludes the proof.

\section{Xi. Appendix E - Proof of Proposition 4}

Proof: The $\Upsilon$ in (14) can be equivalently written as $\Upsilon=\sum_{j=i-k}^{i} \operatorname{Tr}\left[\left(\mathbf{Y}_{j}-\mathbf{Z} \overline{\mathbf{S}}_{j}^{i-k+1}\right)\left(\mathbf{Y}_{j}-\mathbf{Z} \overline{\mathbf{S}}_{j}^{i-k+1}\right)^{H}\right]=$ $\sum_{j=i-k}^{i} \operatorname{Tr}\left(\mathbf{Y}_{j} \mathbf{Y}_{j}^{H}\right)+\operatorname{Tr}\left(\mathbf{Z} \overline{\mathbf{S}}_{j}^{i-k+1} \overline{\mathbf{S}}_{j}^{i-k+1^{H}} \mathbf{Z}^{H}\right)-$ $\operatorname{Tr}\left(\mathbf{Y}_{j} \overline{\mathbf{S}}_{j}^{i-k+1{ }^{H}} \mathbf{Z}^{H}\right)-\operatorname{Tr}\left(\mathbf{Z} \overline{\mathbf{S}}_{j}^{i-k+1} \mathbf{Y}_{j}^{H}\right)$.

By employing the results of [51], we have $\frac{\partial \Upsilon}{\partial \mathbf{Z}}=\sum_{j=i-k}^{i} \mathbf{Z} \overline{\mathbf{S}}_{j}^{i-k+1} \overline{\mathbf{S}}_{j}^{i-k+1^{H}}-\mathbf{Y}_{j} \overline{\mathbf{S}}_{j}^{i-k+1^{H}}$. Changing the variable from $j \rightarrow(i-l)$, we have

\footnotetext{
${ }^{6} \mathrm{Here}$, by worst case we mean a set of transmission blocks that results in maximum number of distinct differential constellation points.
}

$\frac{\partial \Upsilon}{\partial \mathbf{Z}}=\sum_{l=0}^{k} \mathbf{Z} \overline{\mathbf{S}}_{i-l}^{i-k+1} \overline{\mathbf{S}}_{i-l}^{i-k+1^{H}}-\mathbf{Y}_{i-l} \overline{\mathbf{S}}_{i-l}^{i-k+1^{H}}=$ $\mathbf{Z} \sum_{l=0}^{k} \overline{\mathbf{S}}_{i-l}^{i-k+1} \overline{\mathbf{S}}_{i-l}^{i-k+1^{H}}-\sum_{l=0}^{k} \mathbf{Y}_{i-l} \overline{\mathbf{S}}_{i-l}^{i-k+1^{H}}$. This concludes the proof.

\section{REFERENCES}

[1] I. E. Telatar "Capacity of multi-antenna Gaussian channels," Eur. Trans. Telecommun., vol. 10, no. 6, pp. 585-595, Dec. 1999.

[2] P. Wolniansky, G. Foschini, G. Golden and R. Valenzuela, "V-BLAST: an architecture for realizing very high data rates over the rich-scattering wireless channel," in Proc. International Symp. Signals, Syst., Electron., Pisa, Italy, Sep. 1998, pp. 295-300.

[3] D. Feng, C. Jiang, G. Lim, L. J. Cimini, G. Feng and G. Y. Li, "A survey of energy-efficient wireless communications," IEEE Commun. Surveys \& Tutorials, vol. 15, no. 1, pp. 167-178, First Quarter 2013.

[4] G. Y. Li et al., "Energy-efficient wireless communications: tutorial, survey, and open issues," IEEE Wireless Commun., vol. 18, no. 6, pp. 28-35, December 2011.

[5] R. Mesleh, H. Haas, C. Ahn and S. Yun "Spatial modulation - a new low complexity spectral efficiency enhancing technique," in Proc. First International Conf. Commun. Netw., Beijing, China., Oct. 2006, pp. 1-5.

[6] R. Mesleh, H. Haas, S. Sinanovic, C. Ahn and S. Yun "Spatial modulation," IEEE Trans. Veh. Technol., vol. 57, no. 4, pp. 2228-2242, July 2008.

[7] M. Di Renzo, H. Haas and P. M. Grant, "Spatial modulation for multipleantenna wireless systems - A Survey," IEEE Commun. Magazine, vol. 49, no. 12, pp. 182-191, Dec. 2011.

[8] M. Di Renzo, H. Haas, A. Ghrayeb, S. Sugiura, L. Hanzo, "Spatial modulation for generalized MIMO: Challenges, opportunities, and implementation," Proc. of the IEEE, vol. 102, no. 1, pp. 56-103, Jan. 2014

[9] J. Jeganathan, A. Ghrayeb and L. Szczecinski, "Spatial modulation: optimal detection and performance analysis," IEEE Commun. Lett. vol. 12, no. 8, pp. 545-547, Aug. 2008.

[10] S. Sugiura, C. Xu, S. X. Ng and L. Hanzo, "Reduced-complexity coherent versus non-coherent QAM-aided space-time shift keying," IEEE Trans. Commun., vol. 59, no. 11, pp. 3090-3101, Nov. 2011.

[11] J. Wang, S. Jia and J. Song, "Signal vector based detection scheme for spatial modulation," IEEE Commun. Lett., vol. 16, no. 1, pp. 19-21, Jan. 2012.

[12] Q. Tang, Y. Xiao, P. Yang, Q. Yu and S. Li, "A new low-complexity nearML detection algorithm for spatial modulation," IEEE Wireless Comm. Lett., vol. 2, no. 1, pp. 90-93, Feb. 2013.

[13] R. Rajashekar, K.V.S. Hari, L. Hanzo, "Reduced-complexity ML detection and capacity-optimized training for spatial modulation systems," IEEE Trans. Commun., vol. 62, no. 1, pp. 112-125, Jan. 2014

[14] A. Stavridis, S. Sinanovic, M. Di Renzo, H. Haas and P. Grant, "An energy saving base station employing spatial modulation," 2012 IEEE 17th International Workshop on Computer Aided Modeling and Design of Communication Links and Networks (CAMAD), Barcelona, 2012, pp. 231-235.

[15] A. Stavridis, S. Sinanovic, M. Di Renzo and H. Haas, "Energy evaluation of spatial modulation at a multi-antenna base station," 2013 IEEE 78th Vehicular Technology Conference (VTC Fall), Las Vegas, NV, 2013, pp. $1-5$.

[16] E. Basar, U. Aygolu, E. Panayirci and H. V. Poor, "Space-time block coding for spatial modulation," IEEE Trans. Commun., vol. 59, no. 3, pp. 823-832, Mar. 2011.

[17] M. Di Renzo and H. Haas, "Transmit-diversity for spatial modulation (SM): Towards the design of high-rate spatially-modulated space-time block codes," in Proc. IEEE Int. Conf. Commun., June 2011.

[18] R. Rajashekar and K.V.S. Hari, "Modulation diversity for spatial modulation using complex interleaved orthogonal design," in Proc. IEEE TENCON 2012, Nov. 2012, pp. 1-6.

[19] X. Li and L. Wang, "High rate space-time block coded spatial modulation with cyclic structure," IEEE Commun. Lett., vol. 18, no. 4, pp. 532-535, Apr. 2014

[20] A. G. Helmy, M. Di Renzo and N. Al-Dhahir, "Enhanced-reliability cyclic generalized spatial-and-temporal modulation," IEEE Commun. Lett., vol. 20, no. 12, pp. 2374-2377, Dec. 2016.

[21] P. Yang, Y. Xiao, Y. Yu and S. Li, "Adaptive spatial modulation for wireless MIMO transmission systems," IEEE Commun. Lett., vol. 15, no. 6, pp. 602-604, June 2011.

[22] P. Yang, Y. Xiao, L. Li, Q. Tang, Y. Yu and S. Li, "Link adaptation for spatial modulation with limited feedback," IEEE Trans. Veh. Technol., vol. 61, no. 8, pp. 3808-3813, Oct. 2012. 
[23] R. Rajashekar, K.V.S. Hari and L. Hanzo, "Antenna selection in spatial modulation systems," IEEE Commun. Lett., vol. 17, no. 3, pp. 521-524, Mar. 2013

[24] R. Rajashekar, K.V.S. Hari and L. Hanzo, "Quantifying the transmit diversity order of Euclidean distance based antenna selection in spatial modulation," IEEE Signal Proc. Lett., vol. 22, no. 9, pp. 1434-1437, Sept. 2015.

[25] J. Zheng and J. Chen, "Further complexity reduction for antenna selection in spatial modulation systems," IEEE Commun. Lett., vol. 19, no. 6, pp. 937-940, June 2015.

[26] P. Yang, Y. Xiao, Y. L. Guan, S. Li and L. Hanzo, "Transmit antenna selection for multiple-input multiple-output spatial modulation systems," IEEE Trans. on Commun., vol. 64, no. 5, pp. 2035-2048, May 2016.

[27] B. Hassibi and B. M. Hochwald, "How much training is needed in multiple-antenna wireless links?," IEEE. Trans. Inform. Theory, vol. 49, no. 4, pp. 951-963, Apr. 2003.

[28] B. L. Hughes, "Differential space-time modulation," IEEE Trans. Inform. Theory, vol. 46, no. 7, pp. 2567-2578, Nov. 2000.

[29] B. M. Hochwald and W. Sweldens, "Differential unitary space-time modulation,” IEEE Trans. Commun., vol. 48, no. 12, pp. 2041-2052, Dec. 2000.

[30] Y. Bian, X. Cheng, M. Wen, L. Yang, H.V. Poor, and B. Jiao, "Differential spatial modulation," IEEE Trans. Veh. Technol., vol. 64, no. 7, pp. 3262-3268, July 2015.

[31] N. Ishikawa and S. Sugiura, "Unified differential spatial modulation," IEEE Wireless Commun. Lett., vol. 3, no. 4, pp. 337-340, Aug. 2014.

[32] W. Zhang, Q. Yin, and H. Deng, "Differential full diversity spatial modulation and its performance analysis with two transmit antennas," IEEE Commun. Lett., vol. 19, no. 4, pp. 677-680, April 2015.

[33] R. Rajashekar, N. Ishikawa, S. Sugiura, K.V.S. Hari, and L. Hanzo, "Full-diversity dispersion matrices from algebraic field extensions for differential spatial modulation," IEEE Trans. Veh. Technol. vol. 66, no. 1, pp. 385-394, Jan. 2017.

[34] P. A. Martin, "Differential spatial modulation for APSK in time-varying fading channels," IEEE Commun. Lett., vol. 19, no. 7, pp. 1261-1264, July. 2015

[35] J. Liu, L. Dan, P. Yang, L. Xiao, F. Yu, and Y. Xiao, "High-rate APSKaided differential spatial modulation: Design method and performance analysis," IEEE Commun. Lett., vol. 21, no. 1, pp. 168-171, Jan. 2017.

[36] M. Wen, X. Cheng, Y. Bian, and H. V. Poor, "A low-complexity nearML differential spatial modulation detector," IEEE Signal Proc. Lett., vol. 22, no. 11, pp. 1834-1838, Nov. 2015.

[37] J. Li, M. Wen, X. Cheng, Y. Yan, S. Song and M. H. Lee, "Differential spatial modulation with Gray coded antenna activation order," IEEE Commun. Lett., vol. 20, no. 6, pp. 1100-1103, June 2016.

[38] M. Zhang, M. Wen, X. Cheng and L. Yang, "A dual-hop virtual MIMO architecture based on hybrid differential spatial modulation," Trans. Wireless Commun., vol. 15, no. 9, pp. 6356-6370, Sept. 2016.

[39] Fa-Long Luo, "Digital front-end in wireless communications and broadcasting: Circuits and signal processing," Cambridge University Press, 2011.

[40] C. Xu, R. Rajashekar, N. Ishikawa, S. Sugiura and L. Hanzo, "Differential space-time block code: An index shift keying approach", under preparation.

[41] C. Xu, S. X. Ng and L. Hanzo, "Multiple-symbol differential sphere detection and decision-feedback differential detection conceived for differential QAM,' IEEE Trans. Veh. Technol., vol. 65, no. 10, pp. 83458360, Oct. 2016.

[42] N. Jacobson, "Basic algebra I," 2nd ed., New York:W.H. Freeman, 1985.

[43] B. A. Sethuraman and B. Sundar Rajan, "Full-diversity, high-rate spacetime block codes from division algebras," IEEE Trans. Inform. Theory, vol. 49, no. 10, pp. 2596-2616, Oct. 2003.

[44] H. Men and M. Jin, "A low-complexity ML detection algorithm for spatial modulation systems with M PSK constellation," IEEE Commun. Lett., vol. 18, no. 8, pp. 1375-1378, Aug. 2014.

[45] S. M. Alamouti, "A simple transmit diversity technique for wireless communications," IEEE J. Select. Areas Commun., vol. 16, no. 8, pp. 1451-1458, Oct. 1998.

[46] E. G. Larsson and P. Stoica, "Space-time block coding for wireless communications," Cambridge University Press, 2003.

[47] M. O. Damen, A. Tewfik, and J. C. Belfiore, "A construction of a spacetime code based on number theory," IEEE Trans. Inform. Theory, vol. 48, no. 3, pp. 753-760, Mar. 2002.

[48] J. C. Belfiore, G. Rekaya, and E. Viterbo, "The Golden code: a 2x2 full-rate space-time code with non-vanishing determinants," IEEE Trans. Inform. Theory, vol. 51, no. 4, pp. 1432-1436, Apr. 2005.
[49] S. Yang, J. C. Belifiore, G. Rekaya, and B. Othman, "Perfect space-time block codes for parallel MIMO channels," in Proc. Int. Symp. Inform. Theory, pp. 1949-1953, July 2006.

[50] M. R. Bhatnagar, A. Hjorungnes, and L. Song, "Differential coding for non-orthogonal space-time block codes with non-unitary constellations over arbitrarily correlated Rayleigh channels," IEEE Trans. Wireless Commun., vol. 8, pp. 3985-3995, August 2009.

[51] A. Hjorungnes and D. Gesbert, "Complex-valued matrix differentiation: techniques and key results," IEEE Trans. Signal Processing, vol. 55, no. 6, pp. 2740-2746, June 2007.

[52] G. H. Golub and C. F. Van Loan, "Matrix computations," John Hopkins, 3rd Edition, 1996

[53] J. Wang, S. Jia, and J. Song, "Generalised spatial modulation system with multiple active transmit antennas and low complexity detection scheme,' IEEE Trans. Wireless Commun., vol. 11, no. 4, pp. 1605-1615, Apr. 2012.

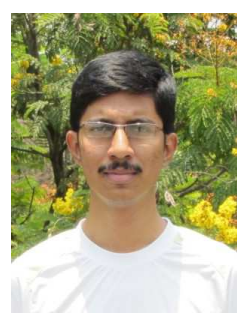

Rakshith Rajashekar received the B.E. degree in electrical communication engineering from Visvesvaraya Technological University, Karnataka, India, in 2007. He received his Ph.D.from the Department of Electrical Communication Engineering, Indian Institute of Science (IISc), India, in 2014. He is presently working as a Research Fellow at the University of Southampton (UoS), UK. Before joining the UoS, he worked at Accord Software \& Systems, Bangalore, India, as a Systems Engineer from 2007 to 2009, and as a Senior Scientist at Broadcom Communications, Bangalore, India from 2014 to 2015. His research interests include antenna selection in MIMO systems, differential communication, millimeter wave communication with a focus on space-time signal processing and coding.

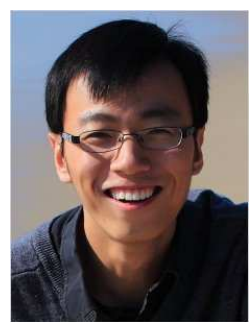

Chao Xu (S'09-M'14) received a B.Eng. degree from Beijing University of Posts and Telecommunications, China, and a BSc(Eng) with First Class Honours from Queen Mary, University of London, UK, through a Sino-UK joint degree program in 2008, both in Telecommunications Enginneering with Management. He obtained a MSc degree with distinction in Radio Frequency Communication Systems and a Ph.D. degree in Wireless Communications from the University of Southampton, UK in 2009 and 2015, respectively. He is currently a postdoctoral researcher working at Southampton Wireless Group, University of Southampton, UK. His research interests include reduced-complexity MIMO design, noncoherent detection, extrinsic-information-transfer-chart-aided turbo detection, and cooperative communications. He was awarded the Best M.Sc. Student in Broadband and Mobile Communication Networks by the IEEE Communications Society (United Kingdom and Republic of Ireland Chapter) in 2009. He also received 2012 Chinese Government Award for Outstanding Self-Financed Student Abroad.

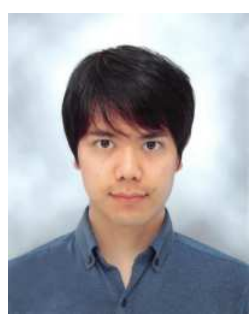

Naoki Ishikawa (S'13) was born in Kanagawa, Japan, in 1991. He received the B.E., M.E., and Ph.D. degrees from the Tokyo University of Agriculture and Technology, Tokyo, Japan, in 2014, 2015, and 2017, respectively. From June 2015 to September 2015, he was an academic visitor with the School of Electronics and Computer Science, University of Southampton, UK. From April 2016 to March 2017, he was a research fellow of the Japan Society for the Promotion of Science. From April 2017, he has been an assistant professor in the Graduate School of Information Sciences, Hiroshima City University, Japan.

He received six domestic student awards, including the Telecom System Technology student Award (honorable mention) from Telecommunications Advancement Foundation of Japan in 2014, the Outstanding Paper Award for Young C\&C Researchers from NEC Computer and Communications Foundation in 2014, the Young Researcher's Encouragement Award from the IEEE VTS Japan Chapter in 2014, and three honors from the Tokyo University of Agriculture and Technology. 


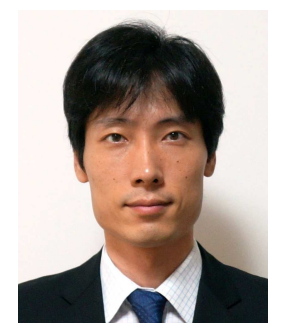

Shinya Sugiura (M'06-SM'12) received the B.S. and M.S. degrees in aeronautics and astronautics from Kyoto University, Kyoto, Japan, in 2002 and 2004, respectively, and the Ph.D. degree in electronics and electrical engineering from the University of Southampton, Southampton, U.K., in 2010.

From 2004 to 2012, he was a Research Scientist with Toyota Central Research and Development Laboratories, Inc., Aichi, Japan. Since 2013, he has been an Associate Professor with the Department of Computer and Information Sciences, Tokyo University of Agriculture and Technology, Tokyo, Japan, where he heads the Wireless Communications Research Group. His research has covered a range of areas in wireless communications, networking, signal processing, and antenna technology. He authored or coauthored over 80 refereed research publications, including 50 IEEE journal and magazine papers.

Dr. Sugiura was a recipient of a number of awards, including the Sixth RIEC Award from the Foundation for the Promotion of Electrical Communication in 2016, the Young Scientists' Prize by the Minister of Education, Culture, Sports, Science and Technology of Japan in 2016, the 14th Funai Information Technology Award (First Prize) from the Funai Foundation in 2015, the 28th Telecom System Technology Award from the Telecommunications Advancement Foundation in 2013, the Sixth IEEE Communications Society Asia-Pacific Outstanding Young Researcher Award in 2011, the 13th Ericsson Young Scientist Award in 2011, and the 2008 IEEE Antennas and Propagation Society Japan Chapter Young Engineer Award. He was also certified as an Exemplary Reviewer of IEEE COMMUNICATIONS LETTERS in 2013 and 2014.

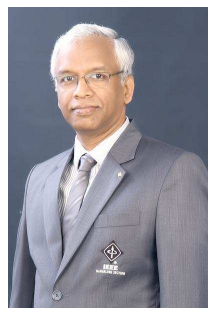

K.V.S. Hari (M'92-SM'97-F'15) received the B.E. degree from Osmania University, Hyderabad, India, in 1983; the M.Tech. degree from the Indian Institute of Technology Delhi, New Delhi, India, in 1985; and the Ph.D. degree from the University of California at San Diego, La Jolla, CA, USA, in 1990.Since 1992, he has been with the Department of Electrical Communication Engineering, Indian Institute of Science, Bangalore, India, where he is currently a Professor and coordinates the activities of the Statistical Signal Processing Laboratory. He was an Affiliated Professor (2010-16) with the Department of Signal Processing, KTH Royal Institute of Technology, Stockholm, Sweden. He has been a Visiting Faculty Member with Stanford University, Stanford, CA, USA; KTH Royal Institute of Technology, Stockholm, Sweden; and Aalto University, Espoo, Finland (formerly Helsinki University of Technology). While at Stanford University, he worked on multiple-input multiple-output (MIMO) wireless channel modelling and co-authored the Worldwide Interoperability for Microwave Access standard on wireless channel models for fixed-broadband wireless communication systems, which proposed the Stanford University Interim channel models. He was also with the Defence Electronics Research Laboratory, Hyderabad, and the Research and Training Unit for Navigational Electronics, Osmania University. His research interests include the development of signal processing algorithms for MIMO wireless communication systems, sparse signal recovery problems, indoor positioning, assistive technologies for the elderly, and neuroscience. Dr. Hari was cu an Editor of Elsevier's EURASIP journal Signal Processing (2006-16) and the Senior Associate Editor of Springer's Indian Academy of Sciences journal SADHANA. He is an Academic Entrepreneur and a Cofounder of the company ESQUBE Communication Solutions, Bangalore. He received the Institution of Electronics and Telecommunication Engineers S. V. C. Aiya Award for Excellence in Telecom Education and the Distinguished Alumnus Award from the Osmania University College of Engineering, Hyderabad. He is a Fellow of the Indian NAE.

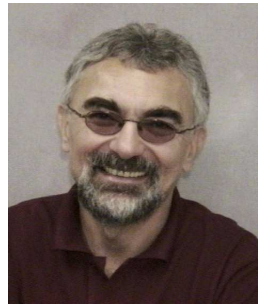

Lajos Hanzo (http://www-mobile.ecs.soton.ac.uk) FREng, FIEEE, FIET, Fellow of EURASIP, DSc received his degree in electronics in 1976 and his doctorate in 1983. In 2009 he was awarded the honorary doctorate "Doctor Honoris Causa" by the Technical University of Budapest. During his 38-year career in telecommunications he has held various research and academic posts in Hungary, Germany and the UK. Since 1986 he has been with the School of Electronics and Computer Science, University of Southampton, UK, where he holds the chair in telecommunications. He has successfully supervised about 100 $\mathrm{PhD}$ students, co-authored 20 John Wiley/IEEE Press books on mobile radio communications totalling in excess of 10,000 pages, published 1,500+ research entries at IEEE Xplore, acted both as TPC and General Chair of IEEE conferences, presented keynote lectures and has been awarded a number of distinctions. Currently he is directing a 100-strong academic research team, working on a range of research projects in the field of wireless multimedia communications sponsored by industry, the Engineering and Physical Sciences Research Council (EPSRC) UK, the European Research Council's Advanced Fellow Grant and the Royal Society's Wolfson Research Merit Award. He is an enthusiastic supporter of industrial and academic liaison and he offers a range of industrial courses.

Lajos is a Fellow of the Royal Academy of Engineering, of the Institution of Engineering and Technology, and of the European Association for Signal Processing. He is also a Governor of the IEEE VTS. During 2008-2012 he was the Editor-in-Chief of the IEEE Press and a Chaired Professor also at Tsinghua University, Beijing. He has 22,000+ citations. For further information on research in progress and associated publications please refer to http://wwwmobile.ecs.soton.ac.uk. 